ESAIM: M2AN 46 (2012) 1363-1387

DOI: $10.1051 / \mathrm{m} 2 \mathrm{an} / 2012006$
ESAIM: Mathematical Modelling and Numerical Analysis

www.esaim-m2an.org

\title{
T-COERCIVITY FOR SCALAR INTERFACE PROBLEMS BETWEEN DIELECTRICS AND METAMATERIALS
}

\author{
Anne-Sophie Bonnet-Ben Dhia ${ }^{1}$, Luchs Chesnel $^{1}$ and Patrick Ciarlet Jr. ${ }^{1}$
}

\begin{abstract}
Some electromagnetic materials have, in a given frequency range, an effective dielectric permittivity and/or a magnetic permeability which are real-valued negative coefficients when dissipation is neglected. They are usually called metamaterials. We study a scalar transmission problem between a classical dielectric material and a metamaterial, set in an open, bounded subset of $\mathbb{R}^{d}$, with $d=2,3$. Our aim is to characterize occurences where the problem is well-posed within the Fredholm (or coercive + compact) framework. For that, we build some criteria, based on the geometry of the interface between the dielectric and the metamaterial. The proofs combine simple geometrical arguments with the approach of $T$-coercivity, introduced by the first and third authors and co-worker. Furthermore, the use of localization techniques allows us to derive well-posedness under conditions that involve the knowledge of the coefficients only near the interface. When the coefficients are piecewise constant, we establish the optimality of the criteria.
\end{abstract}

Mathematics Subject Classification. 35Q60, 35Q61, 35J20.

Received February 1st, 2011. Revised November 2, 2011.

Published online April 11, 2012.

\section{INTRODUCTION}

In electromagnetism, one can model materials that exhibit real-valued strictly negative electric permittivity and/or magnetic permeability, within given frequency ranges. These so-called metamaterials, or left-handed materials, raise unusual questions. Among others, in a domain $\Omega$ of $\mathbb{R}^{d}(d=2,3)$, divided into a classical dielectric material and a metamaterial, proving the existence of electromagnetic fields, and computing them, is a challenging issue (see for instance $[11,19,21,23,24]$ ). For example, let us consider a problem in a twodimensional domain, set in the time-harmonic regime with pulsation $\omega>0$. Then, the transmission problems in the transverse magnetic (TM) and transverse electric (TE) modes can be reduced to scalar problems like

$$
\operatorname{div}(\sigma \nabla u)+\omega^{2} \varsigma u=f \text { in } \Omega,
$$

with a source term $f$, and $(\sigma, \varsigma)$ equal to $\left(\varepsilon^{-1}, \mu\right)$ or $\left(\mu^{-1}, \varepsilon\right)$, where $\varepsilon$ is the dielectric permittivity and $\mu$ is the magnetic permeability, plus boundary conditions. Also, when $(\sigma, \varsigma)=(\varepsilon, 0)$, one models typically electrostatic

\footnotetext{
Keywords and phrases. Metamaterials, interface problem, $T$-coercivity.

1 Laboratoire POEMS, UMR 7231 CNRS/ENSTA/INRIA, ENSTA ParisTech, 32, boulevard Victor, 75739 Paris Cedex 15, France. anne-sophie.bonnet-bendhia@ensta-paristech.fr; lucas.chesnel@ensta-paristech.fr;

patrick.ciarlet@ensta-paristech.fr
} 
fields in two- or three-dimensional domains. Let us mention that the extension to the full Maxwell system of equations, which raises additional difficulties (such as compact imbedding results, $c f .[2,6]$ ), is not treated in this paper.

Mathematically speaking, let $\sigma_{k} \in L^{\infty}\left(\Omega_{k}\right), k=1,2$, be real-valued functions such that

$$
\sigma_{1} \geq c_{1}>0 \text { a.e. in } \Omega_{1} \text { and } \sigma_{2} \leq c_{2}<0 \text { a.e. in } \Omega_{2},
$$

with $c_{k}, k=1,2$, constant numbers. Define $\sigma \in L^{\infty}(\Omega)$ in the following way: $\sigma:=\sigma_{k}$ in $\Omega_{k}, k=1,2$, and consider $\varsigma \in L^{\infty}(\Omega)$. In other words, there is a dielectric material in $\Omega_{1}$, and a metamaterial in $\Omega_{2}$, and we have $\bar{\Omega}=\overline{\Omega_{1}} \cup \overline{\Omega_{2}}\left(\Omega_{1} \cap \Omega_{2}=\emptyset\right)$. We assume that $\Omega, \Omega_{1}$ and $\Omega_{2}$ are domains of $\mathbb{R}^{d}(d=2,3)$. We recall that a domain is an open, bounded and connected subset of $\mathbb{R}^{d}(d=2,3)$ with a Lipschitz boundary.

We supplement the PDE with a homogeneous Dirichlet boundary condition, which writes $u=0$ on $\partial \Omega$. The case of the Neumann boundary condition could be handled similarly. In this setting, the source term $f$ belongs to $H^{-1}(\Omega)$, and solutions $u$ are sought in $H_{0}^{1}(\Omega)$. As the imbedding of $H_{0}^{1}(\Omega)$ into $H^{-1}(\Omega)$ is compact, it is enough to study the principal part of the PDE $u \mapsto \operatorname{div}(\sigma \nabla u)$. Hence, we study the operator $A: u \mapsto-\operatorname{div}(\sigma \nabla u)$ of $\mathcal{L}\left(H_{0}^{1}(\Omega), H^{-1}(\Omega)\right.$ ) (the set of linear continuous mappings from $H_{0}^{1}(\Omega)$ to $H^{-1}(\Omega)$ ), associated with the problem

$$
(\mathscr{P}) \mid \begin{aligned}
& \text { Find } u \in H_{0}^{1}(\Omega) \\
& -\operatorname{div}(\sigma \nabla u)=f \quad \text { such that }
\end{aligned}
$$

Classically, one proves that $u$ is a solution to $(\mathscr{P})$ if, and only if, $u$ solves "Find $u \in H_{0}^{1}(\Omega)$ such that $a(u, v)=$ $l(v)$ for all $v \in H_{0}^{1}(\Omega)$ ", with respectively

$$
a(u, v)=(\sigma \nabla u, \nabla v)_{\Omega}, \quad l(v)=_{H^{-1}(\Omega)}\langle f, v\rangle_{H_{0}^{1}(\Omega)} .
$$

Above, $(\cdot, \cdot)_{\Omega}$ is the usual scalar product of $\left(L^{2}(\Omega)\right)^{d}$, whereas $H^{-1}(\Omega)\langle\cdot, \cdot\rangle_{H_{0}^{1}(\Omega)}$ denotes the duality product between $H^{-1}(\Omega)$ and $H_{0}^{1}(\Omega)$. Of course, because of the sign shift of $\sigma$ across the interface $\Sigma$ dividing $\Omega$, the form $a$ is not coercive over $H_{0}^{1}(\Omega) \times H_{0}^{1}(\Omega)$. In particular, one can not apply the Lax-Milgram theorem.

To overcome this difficulty, one can use the $T$-coercivity approach, introduced in [3]. Note that $T$-coercivity can be seen as a reformulation of the classical inf-sup theory [5], using explicit operators to achieve the inf-sup condition. Let us recall the main features of this method. If there exists an isomorphism $T$ of $H_{0}^{1}(\Omega)$ such that the bilinear form $(u, v) \mapsto a(u, T v)$ is coercive, then the Lax-Milgram theorem now applies. Indeed, the problem "find $u \in H_{0}^{1}(\Omega)$ such that $a(u, T v)=l(T v)$ for all $v \in H_{0}^{1}(\Omega)$ " is well-posed. In addition, because $T$ is an isomorphism of $H_{0}^{1}(\Omega)$, one solves in this way the original problem "find $u \in H_{0}^{1}(\Omega)$ such that $a(u, v)=l(v)$ for all $v \in H_{0}^{1}(\Omega)$ ". Therefore, within this framework, one has to find suitable operators $T$. In [3,26], it is shown that $A$ is actually an isomorphism of $\mathcal{L}\left(H_{0}^{1}(\Omega), H^{-1}(\Omega)\right)$ if $\max \left(\inf _{\Omega_{1}} \sigma_{1} / \sup _{\Omega_{2}}\left|\sigma_{2}\right|, \inf _{\Omega_{2}}\left|\sigma_{2}\right| / \sup _{\Omega_{1}} \sigma_{1}\right)>I_{\Sigma} \geq 1$, where $I_{\Sigma}$ is a constant number, that depends only on the geometry of the interface $\Sigma$ between the dielectric material and the metamaterial. However, the value of $I_{\Sigma}$ is not explicitly provided: indeed, it is defined with the help of the norms of abstract operators. In this paper, we shall complement the results of [3] in two ways. First, we provide some explicit values of the constants. Second, we localize the derivation of the extrema to a neighborhood of the interface $\Sigma$. To achieve those aims, we prove that the problem $(\mathscr{P})$ is well-posed in the sense that the operator $A$ is Fredholm, using simple, geometrically defined, operators $T$ : if uniqueness holds then the problem $(\mathscr{P})$ is well-posed, otherwise a non-trivial, finite dimensional kernel can appear. Let us emphasize that this implies a fortiori that the problem with equation $\operatorname{div}(\sigma \nabla u)+\omega^{2} \varsigma u=f$ in $\Omega$ and boundary conditions is well-posed in the Fredholm sense.

In the case where $\sigma_{1}$ and $\sigma_{2}$ are constant numbers, there exist in the literature at least two other approaches that allow one to tackle problem $(\mathscr{P})$. With the help of integral equations, it was first proven in $[8]$ by Costabel and Stephan that, when the interface $\Sigma$ is smooth (of $\mathscr{C}^{2}$-class), problem $(\mathscr{P})$ is well-posed in the Fredholm sense if, and only if, the contrast $\kappa_{\sigma}:=\sigma_{2} / \sigma_{1}$ is different from -1 . Second, the influence of corners over the interface was specifically studied in [4] (see also [9,22]). The authors proved that, when there is a single corner 
with a right angle on the interface, problem $(\mathscr{P})$, with a right-hand side $f$ in $L^{2}(\Omega)$, is not well-posed in the Fredholm sense if, and only if, $\kappa_{\sigma} \in[-3 ;-1 / 3]$ (similar results can be obtained for any value of the angle). Note that we recover those results within the framework we develop hereafter, with the explicit operators $T$. In this sense, we shall refer to them as optimal results.

The outline is the following. After introducing some notations and proving a preliminary result, we first study elementary cases, in simple geometries of $\mathbb{R}^{2}(d=2)$. Then, we combine those results with a localization technique, to solve the problem $(\mathscr{P})$ in the Fredholm sense, in general geometries of $\mathbb{R}^{2}$, and provide some applications when $\sigma_{k}, k=1,2$, are smooth and/or constants. In particular, we prove that one can obtain a criterion, based only on the values of the contrast on the interface. Also, we investigate cases where the results are negative, that is when problem $(\mathscr{P})$ is not well-posed in the Fredholm sense, in a domain of $\mathbb{R}^{2}$. Last, we provide elements of the approach in a domain of $\mathbb{R}^{3}(d=3)$. We cover in particular the elementary cases, which can not always be reduced to 2D configurations: as an illustrative example, we study the problem set in a domain like Fichera's corner.

\section{NotATions AND A PRELIMINARY RESUlT}

Before we proceed, let us introduce some notations.

Given $\mathcal{O}$ an open set of $\mathbb{R}^{d},(\cdot, \cdot)_{\mathcal{O}}$ denotes the usual scalar products of $L^{2}(\mathcal{O})$ and $\left(L^{2}(\mathcal{O})\right)^{d},\|\cdot\|_{\mathcal{O}}$ the associated norms, $\|\cdot\|_{L^{p}(\mathcal{O})}$ the norm of $L^{p}(\mathcal{O})$ or $\left(L^{p}(\mathcal{O})\right)^{d}(p \in[1, \infty] \backslash\{2\})$, and finally $\|\cdot\|_{H_{0}^{1}(\mathcal{O})}=\|\nabla \cdot\|_{\mathcal{O}}$ the norm of $H_{0}^{1}(\mathcal{O})$ and $\|\cdot\|_{H^{-1}(\mathcal{O})}$ the norm of $H^{-1}(\mathcal{O})$.

The boundaries $\partial \Omega$ and $\partial \Omega_{k}, k=1,2$, are divided as follows: let $\Gamma_{k}:=\partial \Omega \cap \partial \Omega_{k}$, for $k=1,2$. Obviously, the interface $\Sigma$ is such that $\bar{\Sigma}=\overline{\Omega_{1}} \cap \overline{\Omega_{2}} . L^{p}$-norms $(p \in[1, \infty])$ over $\bar{\Sigma}$ are written as above, with $\bar{\Sigma}$ replacing $\mathcal{O}$.

Then, if $v$ is measurable in $\Omega$, we use the notations $v_{k}:=\left.v\right|_{\Omega_{k}}, k=1,2$. Next, we introduce ${ }^{2}$

$$
\sigma_{1}^{+}:=\sup _{\Omega_{1}} \sigma_{1}, \quad \sigma_{2}^{+}:=\sup _{\Omega_{2}}\left|\sigma_{2}\right|, \quad \sigma_{1}^{-}:=\inf _{\Omega_{1}} \sigma_{1} \text { and } \sigma_{2}^{-}:=\inf _{\Omega_{2}}\left|\sigma_{2}\right| .
$$

Whenever applicable, the contrast $\kappa_{\sigma}:=\sigma_{2} / \sigma_{1}$ will be defined over $\bar{\Sigma}$ : for instance as a constant number when $\sigma_{k}, k=1,2$ are constant numbers, or as an element of $\mathscr{C}^{0}(\bar{\Sigma})$ when $\sigma_{k}, k=1,2$ are resp. continuous over $\overline{\Omega_{k}}$, $k=1,2$.

Last, we define the Sobolev spaces

$$
H_{0, \Gamma_{k}}^{1}\left(\Omega_{k}\right):=\left\{\left.v\right|_{\Omega_{k}}, v \in H_{0}^{1}(\Omega)\right\}, k=1,2 .
$$

Let us now prove the result below.

Theorem 2.1. Consider an operator $R_{1} \in \mathcal{L}\left(H_{0, \Gamma_{1}}^{1}\left(\Omega_{1}\right), H_{0, \Gamma_{2}}^{1}\left(\Omega_{2}\right)\right)$ with matching condition $\left.\left(R_{1} u_{1}\right)\right|_{\Sigma}=\left.u_{1}\right|_{\Sigma}$ for all $u_{1} \in H_{0, \Gamma_{1}}^{1}\left(\Omega_{1}\right)$, and define

$$
T_{1} u= \begin{cases}u_{1} & \text { in } \Omega_{1} \\ -u_{2}+2 R_{1} u_{1} & \text { in } \Omega_{2} .\end{cases}
$$

If $\sigma_{1}^{-} / \sigma_{2}^{+}>\left\|R_{1}\right\|^{2}$, then the form a is $T_{1}$-coercive and $A: u \mapsto-\operatorname{div}(\sigma \nabla u)$ is an isomorphism from $H_{0}^{1}(\Omega)$ to $H^{-1}(\Omega)$.

Consider an operator $R_{2} \in \mathcal{L}\left(H_{0, \Gamma_{2}}^{1}\left(\Omega_{2}\right), H_{0, \Gamma_{1}}^{1}\left(\Omega_{1}\right)\right)$ with matching condition $\left.\left(R_{2} u_{2}\right)\right|_{\Sigma}=\left.u_{2}\right|_{\Sigma}$ for all $u_{2} \in$ $H_{0, \Gamma_{2}}^{1}\left(\Omega_{2}\right)$, and define

$$
T_{2} u= \begin{cases}u_{1}-2 R_{2} u_{2} & \text { in } \Omega_{1} \\ -u_{2} & \text { in } \Omega_{2} .\end{cases}
$$

If $\sigma_{2}^{-} / \sigma_{1}^{+}>\left\|R_{2}\right\|^{2}$, then the form a is $T_{2}$-coercive and $A: u \mapsto-\operatorname{div}(\sigma \nabla u)$ is an isomorphism from $H_{0}^{1}(\Omega)$ to $H^{-1}(\Omega)$.

\footnotetext{
${ }^{2}$ Everywhere, we write sup for sup ess, respectively inf for inf ess.
} 
Proof. By construction, $T_{1}$ belongs to $\mathcal{L}\left(H_{0}^{1}(\Omega)\right)$. In addition, one has $T_{1} \circ T_{1}=I d$. In particular, $T_{1}$ is an isomorphism of $H_{0}^{1}(\Omega)$. Let us compute now $a\left(u, T_{1} u\right)$, for $u \in H_{0}^{1}(\Omega)$. With the help of Young's inequality, one can write, for all $\eta>0$,

$$
\begin{aligned}
a\left(u, T_{1} u\right) & =\left(\sigma_{1} \nabla u_{1}, \nabla u_{1}\right)_{\Omega_{1}}+\left(\left|\sigma_{2}\right| \nabla u_{2}, \nabla u_{2}\right)_{\Omega_{2}}-2\left(\left|\sigma_{2}\right| \nabla u_{2}, \nabla\left(R_{1} u_{1}\right)\right)_{\Omega_{2}} \\
& \geq\left(\sigma_{1} \nabla u_{1}, \nabla u_{1}\right)_{\Omega_{1}}+\left(\left|\sigma_{2}\right| \nabla u_{2}, \nabla u_{2}\right)_{\Omega_{2}}-\eta\left(\left|\sigma_{2}\right| \nabla u_{2}, \nabla u_{2}\right)_{\Omega_{2}}-1 / \eta\left(\left|\sigma_{2}\right| \nabla\left(R_{1} u_{1}\right), \nabla\left(R_{1} u_{1}\right)\right)_{\Omega_{2}} \\
& \geq\left(\left(\sigma_{1}-\left\|R_{1}\right\|^{2} \sigma_{2}^{+} / \eta\right) \nabla u_{1}, \nabla u_{1}\right)_{\Omega_{1}}+\left(\left|\sigma_{2}\right|(1-\eta) \nabla u_{2}, \nabla u_{2}\right)_{\Omega_{2}} .
\end{aligned}
$$

As a consequence, if $\sigma_{1}^{-} / \sigma_{2}^{+}>\left\|R_{1}\right\|^{2}$, then there exists $C>0$ such that

$$
C\|u\|_{H_{0}^{1}(\Omega)}^{2} \leq a\left(u, T_{1} u\right), \forall u \in H_{0}^{1}(\Omega) .
$$

In other words, $a$ is $T_{1}$-coercive.

On the other hand, one has $T_{2} \in \mathcal{L}\left(H_{0}^{1}(\Omega)\right)$ and $T_{2} \circ T_{2}=I d$. Given $u \in H_{0}^{1}(\Omega)$, we find for all $\eta>0$,

$$
a\left(u, T_{2} u\right) \geq\left(\sigma_{1}(1-\eta) \nabla u_{1}, \nabla u_{1}\right)_{\Omega_{1}}+\left(\left(\left|\sigma_{2}\right|-\left\|R_{2}\right\|^{2} \sigma_{1}^{+} / \eta\right) \nabla u_{2}, \nabla u_{2}\right)_{\Omega_{2}} .
$$

Therefore, if $\sigma_{2}^{-} / \sigma_{1}^{+}>\left\|R_{2}\right\|^{2}$, then there exists $C>0$ such that

$$
C\|u\|_{H_{0}^{1}(\Omega)}^{2} \leq a\left(u, T_{2} u\right), \forall u \in H_{0}^{1}(\Omega),
$$

i.e. $a$ is $T_{2}$-coercive.

To conclude the proof, we know that there exists an isomorphism $T$ of $H_{0}^{1}(\Omega)$, such that the continuous, bilinear form $(u, v) \mapsto \tilde{a}(u, v)=a(u, T v)$ is coercive over $H_{0}^{1}(\Omega) \times H_{0}^{1}(\Omega)$. Evidently, $v \mapsto \tilde{l}(v)=l(T v)$ is a continuous, linear form over $H_{0}^{1}(\Omega)$. According to Lax-Milgram's theorem, there exists one, and only one, $u \in H_{0}^{1}(\Omega)$ such that $\tilde{a}(u, v)=\tilde{l}(v)$ for all $v \in H_{0}^{1}(\Omega)$, with continuous dependency with respect to the

data $\tilde{l}$. Recall that $T$ is an isomorphism of $H_{0}^{1}(\Omega)$. So, there exists one, and only one, $u \in H_{0}^{1}(\Omega)$ such that $a(u, v)=l(v)$ for all $v \in H_{0}^{1}(\Omega)$, with continuous dependency with respect to the data $l$. We conclude that $A$ is an isomorphism.

In the rest of the paper, $R_{1}$ denotes an operator of $\mathcal{L}\left(H_{0, \Gamma_{1}}^{1}\left(\Omega_{1}\right), H_{0, \Gamma_{2}}^{1}\left(\Omega_{2}\right)\right)$, and $R_{2}$ denotes an operator of $\mathcal{L}\left(H_{0, \Gamma_{2}}^{1}\left(\Omega_{2}\right), H_{0, \Gamma_{1}}^{1}\left(\Omega_{1}\right)\right)$. Also, $T_{1}$ and $T_{2}$ denote the operators of $\mathcal{L}\left(H_{0}^{1}(\Omega)\right)$ respectively defined by $(2.1)$ and (2.2), for operators $R_{1}$ and $R_{2}$ that fulfill the matching conditions.

\section{A STUdy of ELEMENTARY CASES: GLOBAL CONDITIONS}

Let us build explicit operators that ensure $T$-coercivity, on a series of particular geometries. In a second step (see Sect. 4), we shall handle general geometries. The underlying idea is to provide a criterion, based on the values of $\sigma$, that allows one to prove that $A$ is an isomorphism from $H_{0}^{1}(\Omega)$ to $H^{-1}(\Omega)$.

\subsection{Symmetric domain}

Let $\Omega$ be a symmetric domain, in the sense that $\Omega_{1}$ and $\Omega_{2}$ can be mapped from one to the other with the help of a reflection symmetry. Without loss of generality, we assume that the interface $\Sigma$ is included in the line of equation $y=0$ (see Fig. 1 for an example). In this case, we can prove the result below.

Theorem 3.1 (symmetric domain). Assume that

$$
\max \left(\sigma_{1}^{-} / \sigma_{2}^{+}, \sigma_{2}^{-} / \sigma_{1}^{+}\right)>1 .
$$

Then, there exists an isomorphism $T \in \mathcal{L}\left(H_{0}^{1}(\Omega)\right)$ such that the form a is $T$-coercive and $A: u \mapsto-\operatorname{div}(\sigma \nabla u)$ is an isomorphism from $H_{0}^{1}(\Omega)$ to $H^{-1}(\Omega)$. 


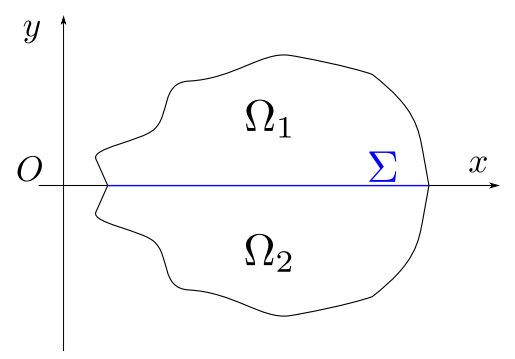

Figure 1. A symmetric geometry.
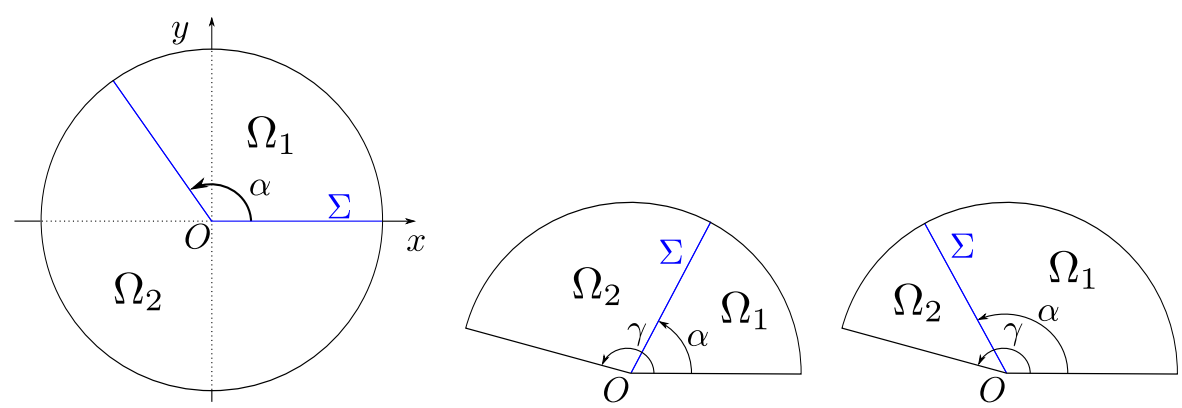

Figure 2. (left) Geometry of an interior vertex. (middle, right) Geometries of a boundary vertex.

Proof. Consider the operators $R_{1}$ and $R_{2}$ respectively defined by $\left(R_{1} u_{1}\right)(x, y)=u_{1}(x,-y)$ and $\left(R_{2} u_{2}\right)(x, y)=$ $u_{2}(x,-y)$. Clearly, one has the matching conditions $\left.\left(R_{k} u_{k}\right)\right|_{\Sigma}=\left.u_{k}\right|_{\Sigma}$ for all $u_{k} \in H_{0, \Gamma_{k}}^{1}\left(\Omega_{k}\right), k=1,2$. Moreover, $\left\|R_{k}\right\|=1$, for $k=1,2$. The conclusion follows from Theorem 2.1 .

Remark 3.2. In the case where $\sigma_{1}$ and $\sigma_{2}$ are constant numbers, Theorem 3.1 shows that $A$ is an isomorphism as soon as the contrast $\kappa_{\sigma}=\sigma_{2} / \sigma_{1}$ is not equal to -1 .

\subsection{Interior vertex}

Consider the geometry of Figure 2-left. More precisely, let us denote by $(r, \theta)$ the polar coordinates centered at $O$ with $\theta=0$ on the half-line $O x$ (positive $x$ ). Given $R>0$ and $0<\alpha<2 \pi$, let us define

$$
\begin{aligned}
& \Omega_{1}:=\{(r \cos \theta, r \sin \theta) \mid 0<r<R, 0<\theta<\alpha\} \\
& \Omega_{2}:=\{(r \cos \theta, r \sin \theta) \mid 0<r<R, \alpha<\theta<2 \pi\} .
\end{aligned}
$$

Theorem 3.3 (interior vertex). Assume that

$$
\max \left(\sigma_{1}^{-} / \sigma_{2}^{+}, \sigma_{2}^{-} / \sigma_{1}^{+}\right)>I_{\alpha}, \quad \text { with } \quad I_{\alpha}:=\max \left(\frac{2 \pi-\alpha}{\alpha}, \frac{\alpha}{2 \pi-\alpha}\right) .
$$

Then, there exists an isomorphism $T \in \mathcal{L}\left(H_{0}^{1}(\Omega)\right)$ such that the form a is $T$-coercive and $A: u \mapsto-\operatorname{div}(\sigma \nabla u)$ is an isomorphism from $H_{0}^{1}(\Omega)$ to $H^{-1}(\Omega)$.

Proof. We keep the same notations for functions expressed either in cartesian coordinates or in polar coordinates. Consider the operators $R_{1}$ and $R_{2}$ respectively defined by $\left(R_{1} u_{1}\right)(\rho, \Theta)=u_{1}\left(\rho, \frac{\alpha}{\alpha-2 \pi}(\Theta-2 \pi)\right)$ and $\left(R_{2} u_{2}\right)(\rho, \Theta)=u_{2}\left(\rho, \frac{\alpha-2 \pi}{\alpha} \Theta+2 \pi\right)$. By construction, one has the matching condition $\left(R_{1} u_{1}\right)(\rho, \alpha)=u_{1}(\rho, \alpha)$ 
and $\left(R_{1} u_{1}\right)(\rho, 2 \pi)=u_{1}(\rho, 0)$, for all $u_{1} \in H_{0, \Gamma_{1}}^{1}\left(\Omega_{1}\right)$. Let us now compute the norm of $R_{1}$. For that, let $u_{1} \in H_{0, \Gamma_{1}}^{1}\left(\Omega_{1}\right)$. Performing the change of variables $(r, \theta)=\left(\rho, \frac{\alpha}{\alpha-2 \pi}(\Theta-2 \pi)\right)$, we find successively

$$
\begin{aligned}
\left\|\nabla\left(R_{1} u_{1}\right)\right\|_{\Omega_{2}}^{2} & =\int_{\Omega_{2}}\left(\frac{\partial\left(R_{1} u_{1}\right)}{\partial \rho}\right)^{2}+\frac{1}{\rho^{2}}\left(\frac{\partial\left(R_{1} u_{1}\right)}{\partial \Theta}\right)^{2} \rho \mathrm{d} \rho \mathrm{d} \Theta \\
& \leq \frac{2 \pi-\alpha}{\alpha} \int_{\Omega_{1}}\left(\frac{\partial u_{1}}{\partial r}\right)^{2} r \mathrm{~d} r \mathrm{~d} \theta+\frac{\alpha}{2 \pi-\alpha} \int_{\Omega_{1}} \frac{1}{r^{2}}\left(\frac{\partial u_{1}}{\partial \theta}\right)^{2} r \mathrm{~d} r \mathrm{~d} \theta \\
& \leq I_{\alpha}\left\|\nabla u_{1}\right\|_{\Omega_{1}}^{2} ; \\
\text { so }\left\|R_{1}\right\|^{2} & \leq I_{\alpha} .
\end{aligned}
$$

Similarly, the matching condition holds for $R_{2}$ on the interface, and $\left\|R_{2}\right\|^{2} \leq I_{\alpha}$. The conclusion follows thanks to Theorem 2.1.

Remark 3.4. One has $-1 \in\left[-I_{\alpha} ;-1 / I_{\alpha}\right]$. Also, if $\alpha=\pi$ this interval reduces to $\{-1\}$, which is consistent with our analysis of symmetric domains (see Sect. 3.1).

Remark 3.5. When $\sigma_{1}$ and $\sigma_{2}$ are constant numbers, Theorem 3.3 implies that $A$ is an isomorphism if $\kappa_{\sigma}=\sigma_{2} / \sigma_{1} \notin\left[-I_{\alpha} ;-1 / I_{\alpha}\right]$. For instance, if $\alpha=\pi / 2$, there holds $\left[-I_{\alpha} ;-1 / I_{\alpha}\right]=[-3 ;-1 / 3]$. So, given $\left.\kappa_{\sigma} \in\right]-\infty ;-3[\cup]-1 / 3 ; 0[$, we know that $A$ is an isomorphism.

Remark 3.6. More generally, one could consider an operator $R_{1}^{\dagger}$ defined by $\left(R_{1}^{\dagger} u_{1}\right)(\rho, \Theta)=u_{1}\left(\rho, g_{1}(\Theta)\right)$ where $g_{1}$ is a $\mathscr{C}^{1}$ diffeomorphism from $[\alpha ; 2 \pi]$ to $[0 ; \alpha]$ such that $g_{1}(2 \pi)=0$ and $g_{1}(\alpha)=\alpha$. Then, one obtains $\left\|R_{1}^{\dagger}\right\|^{2}=\max \left(\left\|g_{1}^{\prime}\right\|_{L^{\infty}([\alpha ; \pi])},\left\|1 /\left(g_{1}^{\prime}\right)\right\|_{L^{\infty}([\alpha ; \pi])}\right)$. According to the mean value theorem, one has $\left\|R_{1}^{\dagger}\right\|^{2} \geq I_{\alpha}$, so our choice $g_{1}(\Theta)=\frac{\alpha}{\alpha-2 \pi}(\Theta-2 \pi)$ is optimal in this configuration.

\subsection{Boundary vertex}

Given $R>0$ and $0<\alpha<\gamma<2 \pi$, let us introduce, with $(r, \theta)$ the polar coordinates defined as before:

$$
\begin{aligned}
& \Omega_{1}:=\{(r \cos \theta, r \sin \theta) \mid 0<r<R, 0<\theta<\alpha\} ; \\
& \Omega_{2}:=\{(r \cos \theta, r \sin \theta) \mid 0<r<R, \alpha<\theta<\gamma\} .
\end{aligned}
$$

Theorem 3.7 (boundary vertex). Assume that

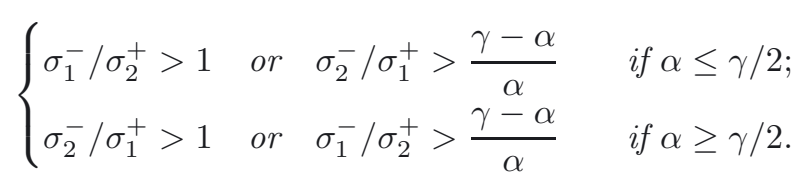

Then, there exists an isomorphism $T \in \mathcal{L}\left(H_{0}^{1}(\Omega)\right)$ such that the form a is $T$-coercive and $A: u \mapsto-\operatorname{div}(\sigma \nabla u)$ is an isomorphism from $H_{0}^{1}(\Omega)$ to $H^{-1}(\Omega)$.

Proof. Let us consider first that $\alpha \leq \gamma / 2$ (Fig. 2-middle), with the operators $R_{1}$ and $R_{2}$, respectively defined by

$$
\left(R_{1} u_{1}\right)(\rho, \Theta)=\left\{\begin{array}{ll}
u_{1}(\rho, 2 \alpha-\Theta) & \text { if } \Theta \leq 2 \alpha \\
0 & \text { else }
\end{array} \quad\left(R_{2} u_{2}\right)(\rho, \Theta)=u_{2}\left(\rho, \frac{\alpha-\gamma}{\alpha} \Theta+\gamma\right) .\right.
$$

One proves the results as before (see Thms. 3.1 (for $R_{1}$ ) and 3.3 (for $R_{2}$ )).

Similarly, one can handle the case where $\alpha \geq \gamma / 2$ (Fig. 2-right).

Remark 3.8. If $\alpha=\gamma / 2$, we recover the result on symmetric domains (see Thm. 3.1).

Remark 3.9. Consider that $\sigma_{1}$ and $\sigma_{2}$ are constant numbers. Then, for instance with $\gamma=\pi$ and $\alpha=\pi / 4$, the previous result indicates that $A$ is an isomorphism, as soon as $\left.\kappa_{\sigma} \in\right]-\infty ;-3[\cup]-1 ; 0[$. 


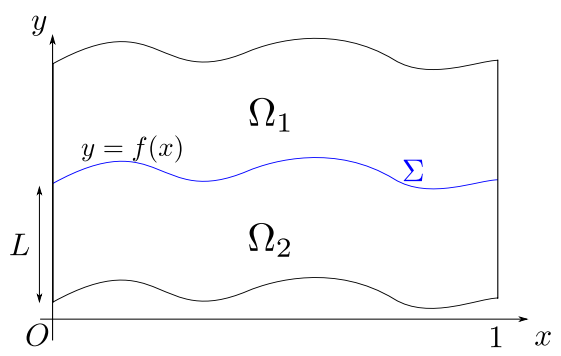

Figure 3. Geometry for an interface of $\mathscr{C}^{1}$-class.

\subsection{Interface of $\mathscr{C}^{1}$-class}

Let us conclude this overview of particular cases with a study of a smooth interface $\Sigma$. Let $f$ be a real-valued function that belongs to $\mathscr{C}^{1}([0 ; 1])$, and let $L>0$. Let us introduce (see Fig. 3 )

$$
\begin{aligned}
& \Omega:=\{(x, y) \mid 0<x<1, f(x)-L<y<f(x)+L\} ; \\
& \Omega_{1}:=\{(x, y) \mid 0<x<1, f(x)<y<f(x)+L\} \\
& \Omega_{2}:=\{(x, y) \mid 0<x<1, f(x)-L<y<f(x)\} .
\end{aligned}
$$

Theorem 3.10. Assume that

$$
\max \left(\sigma_{1}^{-} / \sigma_{2}^{+}, \sigma_{2}^{-} / \sigma_{1}^{+}\right)>\left(1+2\left\|f^{\prime}\right\|_{L^{\infty}(\bar{\Sigma})}+4\left\|f^{\prime}\right\|_{L^{\infty}(\bar{\Sigma})}^{2}\right) .
$$

Then, there exists an isomorphism $T \in \mathcal{L}\left(H_{0}^{1}(\Omega)\right)$ such that the form a is $T$-coercive and $A: u \mapsto-\operatorname{div}(\sigma \nabla u)$ is an isomorphism from $H_{0}^{1}(\Omega)$ to $H^{-1}(\Omega)$.

Proof. Define respectively the operators $R_{1}$ and $R_{2}$ by $\left(R_{1} u_{1}\right)(s, t)=u_{1}(s, 2 f(s)-t)$ and $\left(R_{2} u_{2}\right)(s, t)=$ $u_{2}(s, 2 f(s)-t)$. We note that if $(s, t) \in \Sigma$, then $t=f(s)$ and accordingly $\left(R_{1} u_{1}\right)(s, t)=u_{1}(s, 2 f(s)-t)=u_{1}(s, t)$, for all $u_{1} \in H_{0, \Gamma_{1}}^{1}\left(\Omega_{1}\right)$. Next, let us bound the norm of $R_{1}$. Given $u_{1} \in H_{0, \Gamma_{1}}^{1}\left(\Omega_{1}\right)$ and using the change of variables $(x, y)=(s, 2 f(s)-t)$, we find

$$
\begin{aligned}
\left\|\nabla\left(R_{1} u_{1}\right)\right\|_{\Omega_{2}}^{2} & =\int_{\Omega_{2}}\left(\frac{\partial\left(R_{1} u_{1}\right)}{\partial s}\right)^{2}+\left(\frac{\partial\left(R_{1} u_{1}\right)}{\partial t}\right)^{2} \mathrm{~d} s \mathrm{~d} t \\
& \leq \int_{\Omega_{1}}\left(\frac{\partial u_{1}}{\partial x}+2 f^{\prime}(x) \frac{\partial u_{1}}{\partial y}\right)^{2}+\left(\frac{\partial u_{1}}{\partial y}\right)^{2} \mathrm{~d} x \mathrm{~d} y \\
& \leq \int_{\Omega_{1}}\left(\frac{\partial u_{1}}{\partial x}\right)^{2}+4\left|f^{\prime}(x)\right|\left|\frac{\partial u_{1}}{\partial x}\right|\left|\frac{\partial u_{1}}{\partial y}\right|+4\left|f^{\prime}(x)\right|^{2}\left(\frac{\partial u_{1}}{\partial y}\right)^{2}+\left(\frac{\partial u_{1}}{\partial y}\right)^{2} \mathrm{~d} x \mathrm{~d} y \\
& \leq\left(1+2\left\|f^{\prime}\right\|_{L^{\infty}(\bar{\Sigma})}+4\left\|f^{\prime}\right\|_{L^{\infty}(\bar{\Sigma})}^{2}\right)\left\|\nabla u_{1}\right\|_{\Omega_{1}}^{2} .
\end{aligned}
$$

It follows that $\left\|R_{1}\right\|^{2} \leq\left(1+2\left\|f^{\prime}\right\|_{L^{\infty}(\bar{\Sigma})}+4\left\|f^{\prime}\right\|_{L^{\infty}(\bar{\Sigma})}^{2}\right)$.

Reversing the roles of $\Omega_{1}$ and $\Omega_{2}$, one recovers the matching condition for $R_{2}$, and moreover $\left\|R_{2}\right\|^{2} \leq$ $\left(1+2\left\|f^{\prime}\right\|_{L^{\infty}(\bar{\Sigma})}+4\left\|f^{\prime}\right\|_{L^{\infty}(\bar{\Sigma})}^{2}\right)$.

The conclusion follows from Theorem 2.1 .

Remark 3.11. In the special case where $f^{\prime}$ is uniformly equal to 0 , the domain $\Omega$ is symmetric and the result is identical to the one of Theorem 3.1. 


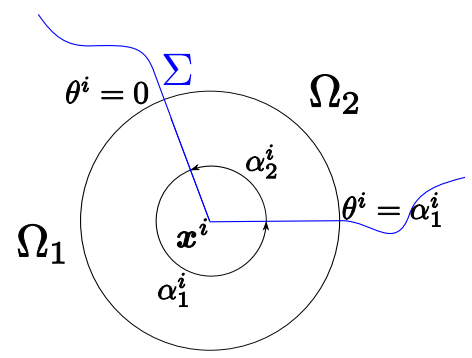

Figure 4. Notations for $\boldsymbol{x}^{i} \in \mathcal{S}_{\mathrm{int}}-\alpha^{i}=\alpha_{2}^{i}$.

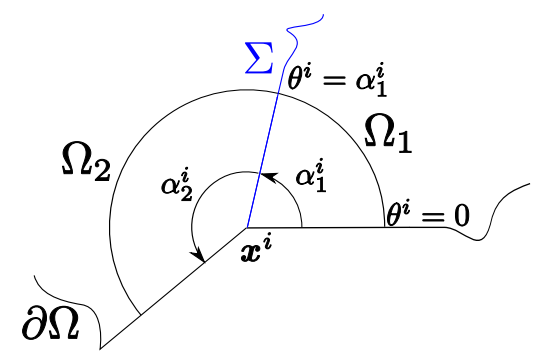

FiguRE 5. Notations for $\boldsymbol{x}^{i} \in \mathcal{S}_{\text {ext }}-\alpha^{i}=\alpha_{1}^{i}$.

\section{A STUdy OF GENERAL GEOMETRIES VIA LOCALIZATION}

The problem $(\mathscr{P})$ is said to be well-posed in the Fredholm sense when the operator $A \in \mathcal{L}\left(H_{0}^{1}(\Omega), H^{-1}(\Omega)\right)$ is Fredholm of index 0. Let us recall the definition below (see for instance [14,25]).

Definition 4.1. Let $X$ and $Y$ be two Banach spaces, and $B$ an operator of $\mathcal{L}(X, Y)$. The operator $B$ is Fredholm if

(i) $\operatorname{dim} \operatorname{ker} B<\infty, \operatorname{Im} B$ is closed;

(ii) $\operatorname{dim} \operatorname{coker} B<\infty$, where coker $B:=Y / \operatorname{Im} B$.

When $B$ is a Fredholm operator, its index is defined by ind $B:=\operatorname{dim} \operatorname{ker} B-\operatorname{dim} \operatorname{coker} B$.

\subsection{Setting of the problem and additional notations}

We recall that $\Omega$ is a domain of $\mathbb{R}^{2}$, that is an open, bounded and connected subset of $\mathbb{R}^{2}$ with a Lipschitz boundary. The domain $\Omega$ is divided into two open subsets $\Omega_{1}$ and $\Omega_{2}$ by an interface $\Sigma$, namely $\overline{\Omega_{1}} \cup \overline{\Omega_{2}}=\bar{\Omega}$, $\Omega_{1} \cap \Omega_{2}=\emptyset$ and $\overline{\Omega_{1}} \cap \overline{\Omega_{2}}=\bar{\Sigma}$. Let $\boldsymbol{n}$ be the unit normal vector to $\Sigma$, going from $\Omega_{1}$ to $\Omega_{2}$. Below, we make a number of regularity assumptions, focusing on the corners and endpoints of the interface (as illustrated by Figs. 4 and 5):

- the subsets $\Omega_{1}$ and $\Omega_{2}$ have a Lipschitz boundary;

- the interface $\Sigma$ is of $\mathscr{C}^{1}$-class, to the exception of a finite number of interior vertices $\mathcal{S}_{\text {int }}=\left\{\boldsymbol{x}^{i}, 1 \leq i \leq N_{\mathrm{int}}\right\}$. And, for $1 \leq i \leq N_{\text {int }}$, the subsets $\Omega_{1}$ and $\Omega_{2}$ coincide with open cones in a neighborhood $\mathcal{V}^{i}$ of $\boldsymbol{x}^{i}$, locally in $\Omega$ :

$$
\begin{aligned}
& \Omega_{1} \cap \mathcal{V}^{i}=\mathcal{K}_{1}\left(\boldsymbol{x}^{i}\right) \cap \mathcal{V}^{i} \text { and } \Omega_{2} \cap \mathcal{V}^{i}=\mathcal{K}_{2}\left(\boldsymbol{x}^{i}\right) \cap \mathcal{V}^{i}, \\
& \text { where } \mathcal{K}_{1}\left(\boldsymbol{x}^{i}\right) \text { and } \mathcal{K}_{2}\left(\boldsymbol{x}^{i}\right) \text { are open cones, centered at } \boldsymbol{x}^{i} ;
\end{aligned}
$$

- there are either 0 or 2 endpoints, called boundary vertices: $\mathcal{S}_{\text {ext }}:=\bar{\Sigma} \cap \partial \Omega=\left\{\boldsymbol{x}^{i}, N_{\text {int }}+1 \leq i \leq N_{\text {int }}+N_{\text {ext }}\right\}$, with $N_{\text {ext }} \in\{0,2\}$. And, for $N_{\text {int }}+1 \leq i \leq N_{\text {int }}+N_{\text {ext }}$, the subsets $\Omega_{1}$ and $\Omega_{2}$ coincide with open cones in a neighborhood $\mathcal{V}^{i}$ of $\boldsymbol{x}^{i}$, locally in $\Omega$ : i.e., (4.1) holds. 
For each index $i$, we define the apertures $\left.\alpha_{k}^{i} \in\right] 0 ; 2 \pi\left[\right.$ of the cones $\mathcal{K}_{k}\left(\boldsymbol{x}^{i}\right), k=1,2$. We introduce $\gamma^{i}:=\alpha_{1}^{i}+\alpha_{2}^{i}$ and $\alpha^{i}:=\min \left(\alpha_{1}^{i}, \alpha_{2}^{i}\right)$. Evidently, one has $\gamma^{i}=2 \pi$ for interior vertices, and $\gamma^{i}<2 \pi$ for boundary vertices. On the other hand, at an interior vertex $\boldsymbol{x}^{i}, \Sigma$ is not of $\mathscr{C}^{1}$-class, so $0<\alpha^{i}<\pi$.

We denote by $\left(r^{i}, \theta^{i}\right)$ the polar coordinates centered at $\boldsymbol{x}^{i}$ with the angle $\theta^{i}$ such that

$$
\begin{aligned}
& \mathcal{K}_{1}\left(\boldsymbol{x}^{i}\right) \text { is isometric to }\left\{\left(r^{i} \cos \theta^{i}, r^{i} \sin \theta^{i}\right) \mid r^{i}>0,0<\theta^{i}<\alpha_{1}^{i}\right\} \\
& \mathcal{K}_{2}\left(\boldsymbol{x}^{i}\right) \text { is isometric to }\left\{\left(r^{i} \cos \theta^{i}, r^{i} \sin \theta^{i}\right) \mid r^{i}>0, \alpha_{1}^{i}<\theta^{i}<\gamma^{i}\right\} .
\end{aligned}
$$

We let $\mathcal{S}_{\text {ext }}^{1}:=\left\{\boldsymbol{x}^{i} \in \mathcal{S}_{\text {ext }} \mid \alpha_{1}^{i} \leq \alpha_{2}^{i}\right\}, \mathcal{S}_{\text {ext }}^{2}:=\left\{\boldsymbol{x}^{i} \in \mathcal{S}_{\text {ext }} \mid \alpha_{2}^{i}<\alpha_{1}^{i}\right\}$ and $\mathcal{S}:=\mathcal{S}_{\text {int }} \cup \mathcal{S}_{\text {ext }}$. The cardinality of $\mathcal{S}$ is denoted by $N$.

Finally, we define

$$
I_{\alpha^{i}}:=\frac{\gamma^{i}-\alpha^{i}}{\alpha^{i}} \text { for } 1 \leq i \leq N
$$

Remark 4.2. Given any interior vertex, there holds $I_{\alpha^{i}}>1$. The same is true for any boundary vertex of $\mathcal{S}_{\text {ext }}^{2}$. On the other hand, for a boundary vertex of $\mathcal{S}_{\text {ext }}^{1}$, one has only $I_{\alpha^{i}} \geq 1$ (it can happen that $I_{\alpha^{i}}=1$ ).

\subsection{Statement of the result}

In our setting, we shall prove that $A$ is Fredholm, under some conditions on the geometry of the domain $\Omega$ and on $\sigma$. Below, we let $B(\boldsymbol{x}, d)$ be the open ball centered at $\boldsymbol{x}$ with radius $d$.

Theorem 4.3. Assume that either 1. or 2. below holds:

$$
\begin{aligned}
\text { 1. } & -\forall \boldsymbol{x} \in \Sigma \backslash \mathcal{S} \text { (smooth part of the interface): } \exists d>0, \inf _{B(\boldsymbol{x}, d) \cap \Omega_{1}} \sigma_{1}>\sup _{B(\boldsymbol{x}, d) \cap \Omega_{2}}\left|\sigma_{2}\right| ; \\
& -\forall \boldsymbol{x}^{i} \in \mathcal{S}_{\text {int }} \cup \mathcal{S}_{\text {ext }}^{2} ; \exists d>0, \inf _{B\left(\boldsymbol{x}^{i}, d\right) \cap \Omega_{1}} \sigma_{1}>I_{\alpha^{i}} \sup _{B\left(\boldsymbol{x}^{i}, d\right) \cap \Omega_{2}}\left|\sigma_{2}\right| ; \\
& -\forall \boldsymbol{x}^{i} \in \mathcal{S}_{\text {ext }}^{1}: \exists d>0, \inf _{B\left(\boldsymbol{x}^{i}, d\right) \cap \Omega_{1}} \sigma_{1}>\sup _{B\left(\boldsymbol{x}^{i}, d\right) \cap \Omega_{2}}\left|\sigma_{2}\right| . \\
\text { 2. } & -\forall \boldsymbol{x} \in \Sigma \backslash \mathcal{S} \text { (smooth part of the interface): } \exists d>0, \inf _{B(\boldsymbol{x}, d) \cap \Omega_{2}}\left|\sigma_{2}\right|>\sup _{B(\boldsymbol{x}, d) \cap \Omega_{1}} \sigma_{1} ; \\
& -\forall \boldsymbol{x}^{i} \in \mathcal{S}_{\text {int }} \cup \mathcal{S}_{\text {ext }}^{1}: \exists d>0, \inf _{B\left(\boldsymbol{x}^{i}, d\right) \cap \Omega_{2}}\left|\sigma_{2}\right|>I_{\alpha^{i}} \sup _{B\left(\boldsymbol{x}^{i}, d\right) \cap \Omega_{1}} \sigma_{1} ; \\
& -\forall \boldsymbol{x}^{i} \in \mathcal{S}_{\mathrm{ext}}^{2}: \exists d>0, \inf _{B\left(\boldsymbol{x}^{i}, d\right) \cap \Omega_{2}}\left|\sigma_{2}\right|>\sup _{B\left(\boldsymbol{x}^{i}, d\right) \cap \Omega_{1}} \sigma_{1} .
\end{aligned}
$$

Then, the operator $A: u \mapsto-\operatorname{div}(\sigma \nabla u)$ of $\mathcal{L}\left(H_{0}^{1}(\Omega), H^{-1}(\Omega)\right)$ is Fredholm of index 0 .

Remark 4.4. Under the assumptions of Theorem 4.3, if $A$ is injective, then $A$ is an isomorphism of $H_{0}^{1}(\Omega)$ into $H^{-1}(\Omega)$. On the other hand, it can happen that the dimension of ker $A$ is finite and not equal to 0 .

The proof is divided in several steps, following Section 5, Chapter 2 of Lions and Magenes [13], Section 6.3 of Kozlov et al. [12] or Section 4.1.2 of Nazarov and Plamenevsky [15]. First, we introduce a partition of unity, which fits the geometry of the domain (and of the interface). Then, we prove an a priori estimate for solutions to $(\mathscr{P})$, with the help of $T$-coercivity. To reach that goal, we use the $T$-coercivity framework that we developed previously on a series of elementary cases. Finally, a classical application of Peetre's lemma leads to the conclusion. 


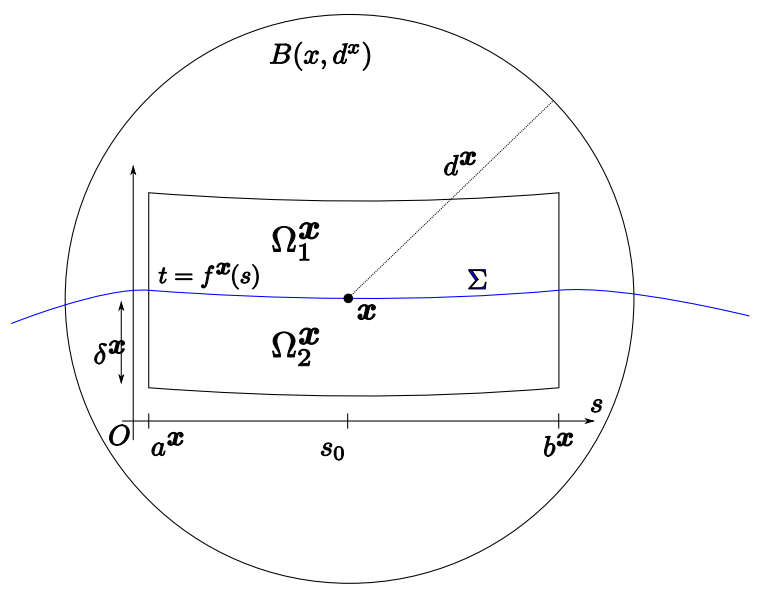

FiguRE 6. Situation in a neighborhood of $\boldsymbol{x}$.

\subsection{Construction of a partition of unity}

Let $\boldsymbol{x}^{i} \in \mathcal{S}$. According to one of the two assumptions (case 1 . or case 2.) of Theorem 4.3, there exists $d^{i}>0$ such that $\left(B\left(\boldsymbol{x}^{i}, d^{i}\right) \cap \Omega\right) \subset \mathcal{V}^{i}$, where $\mathcal{V}^{i}$ is the neighborhood of $\boldsymbol{x}^{i}$ that appears in (4.1), and

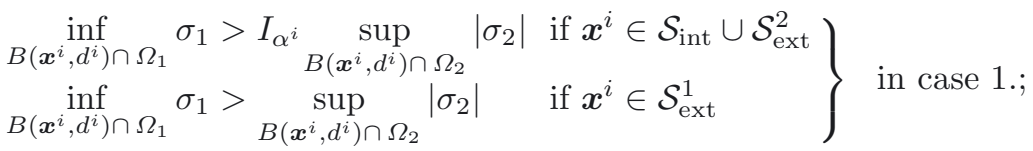

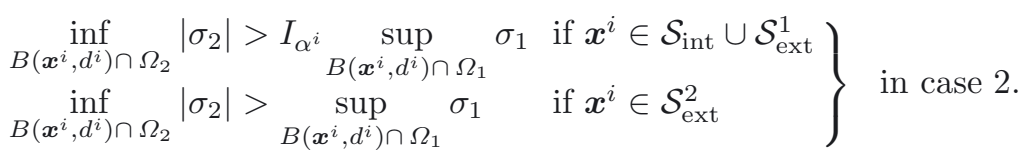

For $1 \leq i \leq N$, let $\zeta^{i} \in \mathscr{C}^{\infty}(\bar{\Omega})$ be a cutoff function, equal to 1 in $\overline{B\left(\boldsymbol{x}^{i}, d^{i} / 2\right)} \cap \Omega$, with support included in $\left(B\left(\boldsymbol{x}^{i}, d^{i}\right) \cap \Omega\right) \subset \mathcal{V}^{i}$, and such that $\zeta^{i}$ is a function of the radius $r^{i}$ only, and $0 \leq \zeta^{i} \leq 1$.

Next, define $\Sigma_{r}:=\Sigma \backslash \bigcup_{i=1}^{N} \overline{B\left(\boldsymbol{x}^{i}, d^{i} / 2\right)}$, and let $\boldsymbol{x} \in \overline{\Sigma_{r}}$. According to the assumption on the smooth part of $\Sigma$, there exists $d^{\boldsymbol{x}}>0$ such that $B\left(\boldsymbol{x}, d^{\boldsymbol{x}}\right) \subset \Omega \backslash \mathcal{S}$, and

$$
\inf _{B\left(\boldsymbol{x}, d^{\boldsymbol{x}}\right) \cap \Omega_{1}} \sigma_{1}>\sup _{B\left(\boldsymbol{x}, d^{\boldsymbol{x}}\right) \cap \Omega_{2}}\left|\sigma_{2}\right| \text { or } \inf _{B\left(\boldsymbol{x}, d^{\boldsymbol{x}}\right) \cap \Omega_{2}}\left|\sigma_{2}\right|>\sup _{B\left(\boldsymbol{x}, d^{\boldsymbol{x}}\right) \cap \Omega_{1}} \sigma_{1} .
$$

On the other hand, as $\Sigma$ is of piecewise $\mathscr{C}^{1}$-class, it coincides locally with the graph of a function $f^{x}$ of $\mathscr{C}^{1}(\mathbb{R})$ (see Annex $\mathrm{C}$ of $[10])$. Let $s_{0} \in \mathbb{R}$ be such that $\boldsymbol{x}=\left(s_{0}, f^{\boldsymbol{x}}\left(s_{0}\right)\right)$. Up to a rotation of the coordinates system, one can assume that $f^{\boldsymbol{x}^{\prime}}\left(s_{0}\right)=0$.

Consider next three real numbers $a^{x}, b^{x}$ and $\delta^{x}>0$ such that the set (as illustrated by Fig. 6)

$$
\Omega^{\boldsymbol{x}}:=\left\{(s, t) \in \mathbb{R}^{2} \mid a^{\boldsymbol{x}}<s<b^{\boldsymbol{x}}, f^{\boldsymbol{x}}(s)-\delta^{\boldsymbol{x}}<t<f^{\boldsymbol{x}}(s)+\delta^{\boldsymbol{x}}\right\}
$$

is included in $B\left(\boldsymbol{x}, d^{\boldsymbol{x}}\right.$ ), and such that $a^{\boldsymbol{x}}<s_{0}<b^{\boldsymbol{x}}$ (so that $\boldsymbol{x}$ belongs to $\Omega^{\boldsymbol{x}}$ ). Choosing the direction of the coordinate axes, one can ensure that $\Omega^{x} \cap \Omega_{1}$ and $\Omega^{x} \cap \Omega_{2}$ coincide respectively with $\Omega_{1}^{x}$ and $\Omega_{2}^{x}$, that are defined by

$$
\begin{aligned}
& \Omega_{1}^{x}:=\left\{(s, t) \in \mathbb{R}^{2} \mid a^{\boldsymbol{x}}<s<b^{\boldsymbol{x}}, f^{\boldsymbol{x}}(s)<t<f^{\boldsymbol{x}}(s)+\delta^{\boldsymbol{x}}\right\} \\
& \Omega_{2}^{\boldsymbol{x}}:=\left\{(s, t) \in \mathbb{R}^{2} \mid a^{\boldsymbol{x}}<s<b^{\boldsymbol{x}}, f^{\boldsymbol{x}}(s)-\delta^{\boldsymbol{x}}<t<f^{\boldsymbol{x}}(s)\right\} .
\end{aligned}
$$


But $f^{\boldsymbol{x} \prime}$ is continuous at $s=s_{0}$ and it vanishes there, so according to (4.2) one can take $a^{\boldsymbol{x}}$ and $b^{\boldsymbol{x}}$ close enough to $s_{0}$ so that

$$
\begin{array}{r}
\inf _{\Omega_{1}^{x}} \sigma_{1}>\sup _{\Omega_{2}^{x}}\left|\sigma_{2}\right|\left(1+2\left\|f^{\prime}\right\|_{L^{\infty}\left(\left[a^{x} ; b^{x}\right]\right)}+4\left\|f^{\prime}\right\|_{L^{\infty}\left(\left[a^{x} ; b^{x}\right]\right)}^{2}\right) \\
\text { or } \inf _{\Omega_{2}^{x}}\left|\sigma_{2}\right|>\sup _{\Omega_{1}^{x}} \sigma_{1}\left(1+2\left\|f^{\prime}\right\|_{L^{\infty}\left(\left[a^{x} ; b^{x}\right]\right)}+4\left\|f^{\prime}\right\|_{L^{\infty}\left(\left[a^{x} ; b^{x}\right]\right)}^{2}\right) .
\end{array}
$$

Consider next

$$
\tilde{\Omega}^{\boldsymbol{x}}:=\left\{(s, t) \in \mathbb{R}^{2} \mid a^{\boldsymbol{x}}+\left(s_{0}-a^{\boldsymbol{x}}\right) / 2<s<b^{\boldsymbol{x}}-\left(b^{\boldsymbol{x}}-s_{0}\right) / 2, f^{\boldsymbol{x}}(s)-\delta^{\boldsymbol{x}} / 2<t<f^{\boldsymbol{x}}(s)+\delta^{\boldsymbol{x}} / 2\right\} .
$$

By construction, $\tilde{\Omega}^{x}$ is a neighborhood of $\boldsymbol{x}$, and $\tilde{\Omega}^{x} \subset \Omega^{x}$.

The set $\overline{\Sigma_{r}}$ is compact, so one can extract from the set $\left(\tilde{\Omega}^{x}\right)_{\boldsymbol{x} \in \overline{\Sigma_{r}}}$ a finite collection, denoted by $\left(\tilde{\mathcal{O}}^{i}\right)_{i=1}^{N_{\Sigma}}$, whose union covers $\overline{\Sigma_{r}}$. Further, for $1 \leq i \leq N_{\Sigma}$, we let $\mathcal{O}^{i}$ denote the open set $\Omega^{x}$ associated with $\tilde{\Omega}^{x}=\tilde{\mathcal{O}}^{i}$. Thus, it is possible to introduce a bounded, open set $\mathcal{O}^{0}$ (of $\mathbb{R}^{2}$ ) which does not intersect $\Sigma$, and such that

$$
\Omega \subset\left(\mathcal{O}^{0} \cup\left(\bigcup_{i=1}^{N_{\Sigma}} \tilde{\mathcal{O}}^{i}\right) \cup\left(\bigcup_{i=1}^{N} B\left(\boldsymbol{x}^{i}, d^{i} / 2\right)\right)\right)
$$

Next, consider

- a function $\chi^{0} \in \mathscr{C}^{\infty}(\bar{\Omega})$ whose support does not intersect $\bar{\Sigma}$, equal to 1 in $\mathcal{O}^{0}$ and such that $0 \leq \chi^{0} \leq 1$;

- for $1 \leq i \leq N_{\Sigma}$, a function $\chi^{i} \in \mathscr{C}^{\infty}(\bar{\Omega})$, whose support is included in $\mathcal{O}^{i}$, equal to 1 in $\tilde{\mathcal{O}}^{i}$ and such that $0 \leq \chi^{i} \leq 1$.

It follows that, for all $\boldsymbol{x} \in \bar{\Omega}$,

$$
\sum_{i=0}^{N_{\Sigma}} \chi^{i}(\boldsymbol{x})+\sum_{i=1}^{N} \zeta^{i}(\boldsymbol{x}) \geq 1 ; \exists i_{0} \text { such that } \chi^{i_{0}}(\boldsymbol{x})=1 \text { or } \zeta^{i_{0}}(\boldsymbol{x})=1 .
$$

\subsection{A priori estimate for solutions to $(\mathscr{P})$}

Given $u \in H_{0}^{1}(\Omega)$, let $f:=A u=-\operatorname{div}(\sigma \nabla u) \in H^{-1}(\Omega)$. Let us prove there exists $C>0$, independent of $u$, such that

$$
\|u\|_{H_{0}^{1}(\Omega)} \leq C\left(\|A u\|_{H^{-1}(\Omega)}+\|u\|_{\Omega}\right) .
$$

For $\chi \in \mathscr{C}^{\infty}(\bar{\Omega})$, define $\operatorname{supp}^{1} \chi:=\{\boldsymbol{x} \in \bar{\Omega} \mid \chi(\boldsymbol{x})=1\}$, so that one can write

$$
\begin{aligned}
\|u\|_{H_{0}^{1}(\Omega)}^{2} & \leq\|u\|_{H_{0}^{1}\left(\operatorname{supp}^{1} \chi^{0}\right)}^{2}+\sum_{i=1}^{N_{\Sigma}}\|u\|_{H_{0}^{1}\left(\operatorname{supp}^{1} \chi^{i}\right)}^{2}+\sum_{i=1}^{N}\|u\|_{H_{0}^{1}\left(\operatorname{supp}^{1} \zeta^{i}\right)}^{2} \\
& \leq\left\|\chi^{0} u\right\|_{H_{0}^{1}(\Omega)}^{2}+\sum_{i=1}^{N_{\Sigma}}\left\|\chi^{i} u\right\|_{H_{0}^{1}(\Omega)}^{2}+\sum_{i=1}^{N}\left\|\zeta^{i} u\right\|_{H_{0}^{1}(\Omega)}^{2} .
\end{aligned}
$$

Then, let us establish estimates for the three terms of the right-hand side of (4.6).

First,

$$
\begin{aligned}
\left\|\chi^{0} u\right\|_{H_{0}^{1}(\Omega)}^{2} & \leq C\left(|\sigma| \nabla\left(\chi^{0} u\right), \nabla\left(\chi^{0} u\right)\right)_{\Omega} \\
& \leq C\left(\left|\left(|\sigma| u \nabla \chi^{0}, \nabla\left(\chi^{0} u\right)\right)_{\Omega}\right|+\left|\left(|\sigma| \nabla u, \nabla\left(\left(\chi^{0}\right)^{2} u\right)\right)_{\Omega}\right|+\left|\left(|\sigma| \nabla u, \chi^{0} u \nabla \chi^{0}\right)_{\Omega}\right|\right) \\
& \leq C\left(\|u\|_{\Omega}\left\|\chi^{0} u\right\|_{H_{0}^{1}(\Omega)}+\|f\|_{H^{-1}(\Omega)}\left\|\left(\chi^{0}\right)^{2} u\right\|_{H_{0}^{1}(\Omega)}+\|u\|_{H_{0}^{1}(\Omega)}\|u\|_{\Omega}\right) \\
& \leq C\left(\|f\|_{H^{-1}(\Omega)}\|u\|_{H_{0}^{1}(\Omega)}+\|u\|_{\Omega}\|u\|_{H_{0}^{1}(\Omega)}\right) .
\end{aligned}
$$


Using the operators $T_{1}$ or $T_{2}$ (see (2.1) or (2.2)) implicitly defined in the proof of Theorem 3.10 over the domain $\operatorname{int}\left(\operatorname{supp} \chi^{i}\right)$ (with a continuation by 0 in $\Omega \backslash \operatorname{supp} \chi^{i}$ ), one gets an operator of $\mathcal{L}\left(H_{0}^{1}(\Omega)\right.$ ), denoted by $T$. Moreover, one finds

$$
\begin{aligned}
\left\|\chi^{i} u\right\|_{H_{0}^{1}(\Omega)}^{2} & \leq C\left|\left(\sigma \nabla\left(\chi^{i} u\right), \nabla\left(T\left(\chi^{i} u\right)\right)\right)_{\Omega}\right| \\
& \leq C\left(\mid\left(\sigma u \nabla \chi^{i}, \nabla\left(T\left(\chi^{i} u\right)\right)_{\Omega}|+|\left(\sigma \nabla u, \nabla\left(\chi^{i} T\left(\chi^{i} u\right)\right)_{\Omega}|+|\left(\sigma \nabla u, T\left(\chi^{i} u\right) \nabla \chi^{i}\right)_{\Omega} \mid\right)\right.\right. \\
& \leq C\left(\|f\|_{H^{-1}(\Omega)}\|u\|_{H_{0}^{1}(\Omega)}+\|u\|_{\Omega}\|u\|_{H_{0}^{1}(\Omega)}\right) .
\end{aligned}
$$

Indeed, the operator $T$ also belongs to $\mathcal{L}\left(L^{2}(\Omega)\right)$.

Along the same lines, one obtains

$$
\left\|\zeta^{i} u\right\|_{H_{0}^{1}(\Omega)}^{2} \leq C\left(\|f\|_{H^{-1}(\Omega)}\|u\|_{H_{0}^{1}(\Omega)}+\|u\|_{\Omega}\|u\|_{H_{0}^{1}(\Omega)}\right)
$$

because the operators $T_{1}$ or $T_{2}$ (see (2.1) or (2.2)) implicitly used in the proofs of Theorems 3.3 and 3.7 (with a continuation by 0 in $\left.\Omega \backslash \operatorname{supp} \zeta^{i}\right)$, all belong to $\mathcal{L}\left(H_{0}^{1}(\Omega)\right)$ and $\mathcal{L}\left(L^{2}(\Omega)\right)$.

Finally, putting together the estimates (4.6)-(4.9), one concludes that the a priori estimate (4.5) holds.

\subsection{Concluding the proof of Theorem 4.3}

Let us recall a classical result, due to Peetre [20] (see also Lem. 5.1 in [13], Chap. 2, or Lem. 3.4.1 in [12]).

Lemma 4.5. Let $X, Y$ and $Z$ be three reflexive Banach spaces, such that $X$ is compactly embedded into $Z$. Let $B \in \mathcal{L}(X, Y)$. Then the assertions below are equivalent:

(i) $\operatorname{dim} \operatorname{ker} B<\infty$, and $\operatorname{Im} B$ is closed in $Y$;

(ii) there exists $C>0$ such that

$$
\|x\|_{X} \leq C\left(\|B x\|_{Y}+\|x\|_{Z}\right), \forall x \in X
$$

On the one hand, $H_{0}^{1}(\Omega)$ is compactly embedded into $L^{2}(\Omega)$, because $\Omega$ is a bounded subset of $\mathbb{R}^{d}$. On the other hand, coker $A$ is isomorphic to ker $A$ (cf. [14], Thm. 2.13). So Theorem 4.3 follows from Lemma 4.5, (4.5), and ind $A=\operatorname{dim} \operatorname{ker} A-\operatorname{dim} \operatorname{coker} A=0$.

\section{Applichtions}

\subsection{Case of smooth coefficients}

In the case where $\sigma_{k} \in \mathscr{C}^{0}\left(\overline{\Omega_{k}}\right), k=1,2$, the statement of Theorem 4.3 can be simplified. The contrast $\kappa_{\sigma}=\sigma_{2} / \sigma_{1}$ is considered here as an element of $\mathscr{C}^{0}(\bar{\Sigma})$.

Theorem 5.1 (continuous coefficients). Assume that

$$
\text { either }\left\{\begin{array} { l } 
{ \forall \boldsymbol { x } \in \Sigma \backslash \mathcal { S } ( \text { smooth part of } \Sigma ) , \kappa _ { \sigma } ( \boldsymbol { x } ) < - 1 } \\
{ \forall \boldsymbol { x } ^ { i } \in \mathcal { S } _ { \text { int } } \cup \mathcal { S } _ { \text { ext } } ^ { 2 } , \kappa _ { \sigma } ( \boldsymbol { x } ) < - I _ { \alpha ^ { i } } } \\
{ \forall \boldsymbol { x } ^ { i } \in \mathcal { S } _ { \text { ext } } ^ { 1 } , \kappa _ { \sigma } ( \boldsymbol { x } ) < - 1 , }
\end{array} \text { or } \quad \left\{\begin{array}{l}
\forall \boldsymbol{x} \in \Sigma \backslash \mathcal{S}(\text { smooth part of } \Sigma), \kappa_{\sigma}(\boldsymbol{x})>-1 \\
\forall \boldsymbol{x}^{i} \in \mathcal{S}_{\text {int }} \cup \mathcal{S}_{\text {ext }}^{1}, \kappa_{\sigma}(\boldsymbol{x})>-1 / I_{\alpha^{i}} \\
\forall \boldsymbol{x}^{i} \in \mathcal{S}_{\text {ext }}^{2}, \kappa_{\sigma}(\boldsymbol{x})>-1
\end{array}\right.\right.
$$

Then, the operator $A: u \mapsto-\operatorname{div}(\sigma \nabla u)$ of $\mathcal{L}\left(H_{0}^{1}(\Omega), H^{-1}(\Omega)\right)$ is Fredholm of index 0 . 


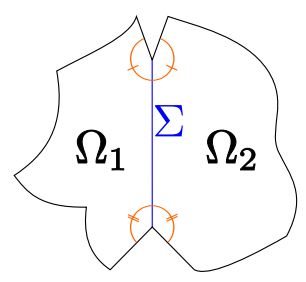

Figure 7. $A$ is Fredholm of index 0 when $\sigma_{2} / \sigma_{1} \in \mathbb{R}_{-}^{*} \backslash\{-1\}$.

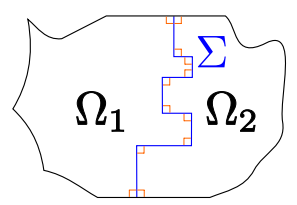

Figure 8. $A$ is Fredholm of index 0 when $\sigma_{2} / \sigma_{1} \in \mathbb{R}_{-}^{*} \backslash[-3 ;-1 / 3]$.

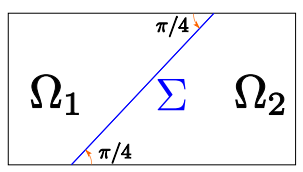

Figure 9. $A$ is Fredholm of index 0 when $\sigma_{2} / \sigma_{1} \in \mathbb{R}_{-}^{*} \backslash[-3 ;-1 / 3]$.

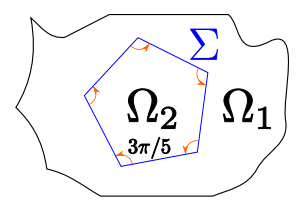

Figure 10. $A$ is Fredholm of index 0 when $\sigma_{2} / \sigma_{1} \in \mathbb{R}_{-}^{*} \backslash[-7 / 3 ;-3 / 7]$.

\subsection{Case of constant coefficients}

When in addition $\sigma_{k}, k=1,2$, are constant numbers, define

$$
\hat{R}_{\Sigma}:=\max \left(\max _{\boldsymbol{x}^{i} \in \mathcal{S}_{\mathrm{int}} \cup \mathcal{S}_{\text {ext }}^{1}} I_{\alpha^{i}}, 1\right), \quad \check{R}_{\Sigma}:=\max \left(\max _{\boldsymbol{x}^{i} \in \mathcal{S}_{\mathrm{int}} \cup \mathcal{S}_{\text {ext }}^{2}} I_{\alpha^{i}}, 1\right) .
$$

There holds the

Theorem 5.2 (constant coefficients). Assume that $\sigma_{2} / \sigma_{1} \in \mathbb{R}_{-}^{*} \backslash\left[-\hat{R}_{\Sigma} ;-1 / \check{R}_{\Sigma}\right]$. Then, the operator $A: u \mapsto$ $-\operatorname{div}(\sigma \nabla u)$ of $\mathcal{L}\left(H_{0}^{1}(\Omega), H^{-1}(\Omega)\right)$ is Fredholm of index 0.

Remark 5.3. With the help of Lax-Milgram's theorem, one proves easily that the operator $A$ is an isomorphism of $\mathcal{L}\left(H_{0}^{1}(\Omega), H^{-1}(\Omega)\right)$ when $\kappa_{\sigma} \in \mathbb{C} \backslash \mathbb{R}_{-}$. One concludes that, when $\sigma_{2} / \sigma_{1} \in \mathbb{C}^{*} \backslash\left[-\hat{R}_{\Sigma} ;-1 / \check{R}_{\Sigma}\right]$, the operator $A$ is Fredholm of index 0 .

We provide now some "practical" illustrations of these results in Figures 7-10.

Remark 5.4. Problems with the Neumann boundary condition can be handled similarly, with the operator $u \mapsto-\operatorname{div}(\sigma \nabla u)$ of $\mathcal{L}\left(H^{1}(\Omega) / \mathbb{R},\left(H^{1}(\Omega) / \mathbb{R}\right)^{\prime}\right)$. In this case, results like those of Theorems 5.1 and 5.2 hold, 
possibly with different bounds on the contrast. So, for the two problems governing the TE and the TM modes to be simultaneously well-posed in the Fredholm sense, one needs that both $\kappa_{\epsilon}$ and $\kappa_{\mu}$ fulfill ad hoc conditions (cf. Thms. 5.1 or 5.2 for $\kappa_{\epsilon}$, and the results with the Neumann boundary condition for $\kappa_{\mu}$ ).

\section{Discussion on the ASSUMPtions ON $\sigma$}

In this section, we establish some results on the operator $A: u \mapsto-\operatorname{div}(\sigma \nabla u)$ of $\mathcal{L}\left(H_{0}^{1}(\Omega), H^{-1}(\Omega)\right)$, in the case where $\sigma$ does not fulfill all the assumptions of Theorem 4.3. We use the contrast $\kappa_{\sigma}=\sigma_{2} / \sigma_{1}$ when $\sigma_{k}, k=1,2$ are constant numbers. Loosely speaking, on a straight part of the interface for which $\kappa_{\sigma}=-1$, we will establish that the operator $A$ is not Fredholm, because of a linear singularity distribution. Indeed, we shall prove that, at any point $\boldsymbol{x}_{0}$ of the (open) straight part of $\Sigma$, one can build a sequence of functions $\left(u_{n}\right)_{n}$ that prevents $A$ from being a Fredholm operator (see Thm. 6.2 below). On the other hand, if $\kappa_{\sigma} \neq-1$, the operator $A$ is not Fredholm if there exist pointwise singularities, located at interior and/or boundary vertices of the interface. This situation happens for values of the contrast lying in an interval (see Thm. 6.4 below). In this latter case, let us mention that Fredholm well-posedness can be recovered in another functional framework [1]. More exotic situations are investigated in Section 6.4.

\subsection{Case of the symmetric domain}

Below, $\Omega$ is a symmetric domain.

Theorem 6.1 (symmetric domain and constant coefficients). Assume that

- $\kappa_{\sigma} \neq-1$ : then $A$ is an isomorphism;

- $\kappa_{\sigma}=-1$ : then $A$ is not a Fredholm operator $(\operatorname{dim} \operatorname{ker} A=\infty)$.

Proof. We consider without loss of generality that the interface $\Sigma$ is included in the line of equation $y=0$ (see Fig. 1).

Theorem 3.1 proves the result when $\kappa_{\sigma} \neq-1$.

Next, consider that $\kappa_{\sigma}=-1$. In this case, we prove that $\operatorname{ker} A$ is an infinite dimensional vector space. To that aim, let $g \in H_{00}^{1 / 2}(\Sigma)$, i.e. $g$ is an element of $H^{1 / 2}(\Sigma)$ such that its continuation by 0 to the whole line of equation $y=0$ belongs to $H^{1 / 2}(\mathbb{R})$. For $k=1,2$, consider then $u_{k} \in H_{0, \Gamma_{k}}^{1}\left(\Omega_{k}\right)$ such that

$$
\begin{cases}\Delta u_{k}=0 & \text { in } \Omega_{k} \\ u_{k}=0 & \text { on } \Gamma_{k} \\ u_{k}=g & \text { on } \Sigma .\end{cases}
$$

By the uniqueness of the solution, we find that $u_{2}(x, y)=u_{1}(x,-y)$ a.e. in $\Omega_{2}$, and it follows that

$$
\sigma_{1} \partial_{\boldsymbol{n}} u_{1}-\sigma_{2} \partial_{\boldsymbol{n}} u_{2}=-\sigma_{1}\left(\partial_{y} u_{1}+\partial_{y} u_{2}\right)=0 \text { a.e. on } \Sigma \text {. }
$$

Summing up, the element $u$ of $H_{0}^{1}(\Omega)$ defined by $u_{\mid \Omega_{k}}=u_{k}$ for $k=1,2$ satisfies $\operatorname{div}(\sigma \nabla u)=0$ in $\Omega$, and as a consequence $A u=0$. As $H_{00}^{1 / 2}(\Sigma)$ is an infinite dimensional vector space, the same is true for $\operatorname{ker} A$.

\subsection{Locally straight interface and contrast equal to -1}

Here, $\Omega$ is a domain of $\mathbb{R}^{2}$ which fulfills the assumptions of Section 4.1.

Theorem 6.2 (locally straight interface and constant coefficients). Assume that $\kappa_{\sigma}=-1$, and that there is an open part of $\Sigma$ which is straight. Then $A$ is not a Fredholm operator.

Remark 6.3. The result remains true, assuming only that $\sigma_{1}$ and $\sigma_{2}$ are locally constant, and take opposite values, in a neighborhood of the straight part of $\Sigma$. 
Proof. According to Lemma 4.5, if $A$ is a Fredholm operator, then there exists $C>0$ such that

$$
\|u\|_{H_{0}^{1}(\Omega)} \leq C\left(\|A u\|_{H^{-1}(\Omega)}+\|u\|_{\Omega}\right), \forall u \in H_{0}^{1}(\Omega) .
$$

Classically, following Hadamard's example, we contradict (6.1) (see also [16,17]).

Let $\boldsymbol{x}_{0}$ be a point on the (open) straight part of $\Sigma$. Up to a rotation of the coordinates system, we can assume that $\Sigma$ is locally included in the line of equation $s=0$, around $\boldsymbol{x}_{0}$. Next, let $b>0$ be sufficiently small, so that $D:=]-b ; b[\times]-b ; b[\subset \Omega$. For $n \in \mathbb{N}$, define

$$
u_{n}(s, t):= \begin{cases}\frac{\sinh n(b+s) \sin n t}{\mathrm{e}^{n b}} & \text { in }[-b ; 0] \times[-b ; b] ; \\ \frac{\sinh n(b-s) \sin n t}{\mathrm{e}^{n b}} & \text { in }[0 ; b] \times[-b ; b] .\end{cases}
$$

Let $\chi_{0} \in \mathscr{C}_{0}^{\infty}(\mathbb{R})$ be an even cutoff function, equal to 1 in a neighborhood of 0 , with support included in ]-b; $b$, and $0 \leq \chi_{0} \leq 1$. Now, let $\chi(s, t):=\chi_{0}(s) \chi_{0}(t)$. Then, the continuation of $\chi u_{n}$ by 0 to $\Omega$, still denoted by $\chi u_{n}$, belongs to $H_{0}^{1}(\Omega)$. We prove now the estimate below, with $C$ independent of $n$ :

$$
\left\|A\left(\chi u_{n}\right)\right\|_{H^{-1}(\Omega)} \leq C\left(\left\|A u_{n}\right\|_{H^{-1}(D)}+\left\|u_{n}\right\|_{D}\right) .
$$

Recall that

$$
\left\|A\left(\chi u_{n}\right)\right\|_{H^{-1}(\Omega)}=\sup _{v \in H_{0}^{1}(\Omega),\|v\|_{H_{0}^{1}(\Omega)}=1}\left|\left(\sigma \nabla\left(\chi u_{n}\right), \nabla v\right)_{\Omega}\right| .
$$

On the other hand, given $v \in H_{0}^{1}(\Omega)$, one has

$$
\left(\sigma \nabla\left(\chi u_{n}\right), \nabla v\right)_{\Omega}=\left(\sigma \nabla u_{n}, \nabla(\chi v)\right)_{\Omega}+\left(\sigma u_{n} \nabla \chi, \nabla v\right)_{\Omega}-\left(\nabla u_{n}, \sigma v \nabla \chi\right)_{\Omega} .
$$

Consider next each term of the right-hand side of (6.4) separately.

- First term:

$$
\left|\left(\sigma \nabla u_{n}, \nabla(\chi v)\right)_{\Omega}\right| \leq C\left\|\operatorname{div}\left(\sigma \nabla u_{n}\right)\right\|_{H^{-1}(D)}\|v\|_{H_{0}^{1}(\Omega)} .
$$

- Second term, using Cauchy-Schwarz inequality:

$$
\left|\left(\sigma u_{n} \nabla \chi, \nabla v\right)_{\Omega}\right| \leq C\left\|u_{n}\right\|_{D}\|v\|_{H_{0}^{1}(\Omega)} .
$$

- Third term, integrated by parts:

$$
\left(\nabla u_{n}, \sigma v \nabla \chi\right)_{\Omega}=\left(u_{n}, \operatorname{div}(\sigma v \nabla \chi)\right)_{D} .
$$

Note that $\operatorname{div}(\sigma v \nabla \chi)$ belongs to $L^{2}(\Omega)$ (and so to $L^{2}(D)$ ), because one has $\left.\sigma v \nabla \chi\right|_{\Omega_{1}} \in H^{1}\left(\Omega_{1}\right),\left.\sigma v \nabla \chi\right|_{\Omega_{2}} \in$ $H^{1}\left(\Omega_{2}\right)$, and finally $\partial_{\boldsymbol{n}} \chi=0$ on $\Sigma$. In addition, $\|\operatorname{div}(\sigma v \nabla \chi)\|_{D} \leq C\|v\|_{H_{0}^{1}(\Omega)}$. Therefore, (6.7) yields

$$
\left|\left(\nabla u_{n}, \sigma v \nabla \chi\right)_{\Omega}\right| \leq C\left\|u_{n}\right\|_{D}\|v\|_{H_{0}^{1}(\Omega)} .
$$

Adding up (6.5), (6.6) and (6.8) to bound the left-hand side of (6.4) leads to (6.3).

On the other hand, one can check by direct inspection that $A u_{n}=0$ in $D$. Indeed, on $]-b ; 0[\times]-b ; b[$ and respectively on $] 0 ; b[\times]-b ; b\left[\right.$, there holds $\Delta u_{n}=0$. Also, on the straight part of the interface, the traces of $u_{n}$ match. Then, as $u_{n}$ is symmetric with respect to the interface and as the contrast is equal to -1 , this implies that the fluxes $\sigma \partial_{n} u_{n}$ also match.

Next, $\left\|u_{n}\right\|_{D} \leq 2 b\left\|u_{n}\right\|_{L^{\infty}(D)}<C$, with $C$ independent of $n$. Consequently, according to (6.3), $\left(A\left(\chi u_{n}\right)\right)_{n \in \mathbb{N}^{*}}$ is bounded in $H^{-1}(\Omega)$. But one can check, again by direct inspection ( $c f$. Lem. 8.1), that

$$
\left\|\chi u_{n}\right\|_{H_{0}^{1}(\Omega)} \underset{n \rightarrow+\infty}{\longrightarrow}+\infty .
$$

This contradicts (6.1), which ends the proof. 


\subsection{Criterion at vertices}

Here, $\Omega$ is a domain of $\mathbb{R}^{2}$ which fulfills the assumptions of Section 4.1.

Theorem 6.4 (vertex and constant coefficients). Assume that either 1., 2. or 3. below holds:

1. there exists $\boldsymbol{x}^{i} \in \mathcal{S}_{\mathrm{int}}$ such that $\left.\kappa_{\sigma} \in\right]-I_{\alpha^{i}} ;-1 / I_{\alpha^{i}}[$;

2. there exists $\boldsymbol{x}^{i} \in \mathcal{S}_{\text {ext }}^{1}$ such that $\left.\left.\kappa_{\sigma} \in\right]-I_{\alpha^{i}} ;-1\right]$;

3. there exists $\boldsymbol{x}^{i} \in \mathcal{S}_{\text {ext }}^{2}$ such that $\kappa_{\sigma} \in\left[-1 ;-1 / I_{\alpha^{i}}[\right.$.

Then the operator $A: u \mapsto-\operatorname{div}(\sigma \nabla u)$ of $\mathcal{L}\left(H_{0}^{1}(\Omega), H^{-1}(\Omega)\right)$ is not Fredholm.

Remark 6.5. If there exists $\boldsymbol{x}^{i} \in \mathcal{S}_{\text {int }} \cup \mathcal{S}_{\text {ext }}^{1}$ such that $\kappa_{\sigma}=-I_{\alpha^{i}}$ or if there exists $\boldsymbol{x}^{i} \in \mathcal{S}_{\text {int }} \cup \mathcal{S}_{\text {ext }}^{2}$ such that $\kappa_{\sigma}=-1 / I_{\alpha^{i}}$, a logarithmic singularity appears (instead of a singularity like $r^{i \eta}$ below). Consequently, we conjecture that the operator $A$ is not Fredholm in those cases.

Proof. Let us focus on the proof of case 1 . In the rest of the proof, we omit the index ${ }^{i}$. If $\kappa_{\sigma}=-1$, Theorem 6.2 allows us to show that $A$ is not Fredholm. Now, assume that $\left.\kappa_{\sigma} \in\right]-I_{\alpha^{i}} ;-1 / I_{\alpha^{i}} \llbracket \backslash\{-1\}$ : we prove in this case that (6.1) cannot hold, using a classical idea in the theory of elliptic operators in non-smooth domains (see for instance part $\mathrm{V}$ of the proof of Thm. 1.2 of [15], p. 104, or Lem. 6.3.3 of [12]). For a value of the contrast lying in $]-I_{\alpha^{i}} ;-1 / I_{\alpha^{i}} \backslash \backslash\{-1\}$, one can show that (follow Sect. 7.3.3 of [22]) there exists a singular function $S(r, \theta)=r^{i \eta} \varphi(\theta)$, with $\eta \in \mathbb{R}^{*}$ and $\varphi$ piecewise $\operatorname{smooth}^{3}$, such that $\operatorname{div}(\sigma \nabla S)=0$. This singular function belongs to $L^{2}(\Omega)$, but not to $H^{1}(\Omega)$. Introduce next a cut-off function $\chi \in \mathscr{C}^{\infty}\left(\mathbb{R}_{+}\right)$, such that $\chi(r)=1$ for $r<d / 2$ and $\chi(r)=0$ for $r>d$, with $d=d^{i}$ of Section 4.3. Define finally $S_{n}(r, \theta):=r^{i \eta+1 / n} \varphi(\theta)$ and $u_{n}(r, \theta):=\chi(r) S_{n}(r, \theta)$. By construction, for $n \in \mathbb{N}^{*}, u_{n}$ belongs to $H_{0}^{1}(\Omega)$, and, according to Lemma 8.2,

$$
\exists C>0, \forall n,\left\|u_{n}\right\|_{\Omega}<C \text { and }\left\|u_{n}\right\|_{H_{0}^{1}(\Omega)} \underset{n \rightarrow+\infty}{\longrightarrow}+\infty .
$$

To contradict (6.1), there remains to prove that the sequence $\left(\operatorname{div}\left(\sigma \nabla u_{n}\right)\right)_{n \in \mathbb{N}^{*}}$ is bounded in $H^{-1}(\Omega)$, which is the more involved part of the proof.

Define $H_{0 \star}^{1}(\Omega):=\left\{u \in H_{0}^{1}(\Omega) \mid u=0\right.$ in a neighbourhood of $\left.\boldsymbol{x}^{i}\right\}$. Since $H_{0 \star}^{1}(\Omega)$ is dense in $H_{0}^{1}(\Omega)$ (see Lem. 1.2.2 in [7]), one has

$$
\left\|\operatorname{div}\left(\sigma \nabla u_{n}\right)\right\|_{H^{-1}(\Omega)}=\sup _{v \in H_{0 \star}^{1}(\Omega),\|v\|_{H_{0}^{1}(\Omega)}=1}\left|\left(\sigma \nabla u_{n}, \nabla v\right)_{\Omega}\right| .
$$

As before, let us write

$$
\begin{aligned}
\left(\sigma \nabla u_{n}, \nabla v\right)_{\Omega} & =\left(\sigma S_{n} \nabla \chi, \nabla v\right)_{\Omega}-\left(\nabla S_{n}, \sigma v \nabla \chi\right)_{\Omega}+\left(\sigma \nabla S_{n}, \nabla(\chi v)\right)_{\Omega} \\
& =\left(\sigma S_{n} \nabla \chi, \nabla v\right)_{\Omega}+\left(S_{n}, \operatorname{div}(\sigma v \nabla \chi)\right)_{\Omega}-\left(\operatorname{div}\left(\sigma \nabla S_{n}\right), \chi v\right)_{\Omega} .
\end{aligned}
$$

Notice that $\operatorname{div}(\sigma v \nabla \chi)$ belongs to $L^{2}(\Omega)$ because one has $\left.\sigma v \nabla \chi\right|_{\Omega_{1}} \in H^{1}\left(\Omega_{1}\right),\left.\sigma v \nabla \chi\right|_{\Omega_{2}} \in H^{1}\left(\Omega_{2}\right)$ and $\partial_{\boldsymbol{n}} \chi=0$ on $\Sigma$. In addition, one checks easily that

$$
\left|\left(\sigma S_{n} \nabla \chi, \nabla v\right)_{\Omega}+\left(S_{n}, \operatorname{div}(\sigma v \nabla \chi)\right)_{\Omega}\right| \leq C\left\|S_{n}\right\|_{\Omega}\|v\|_{H_{0}^{1}(\Omega)} .
$$

Now, let us study the third term of the right-hand side of (6.10). By a direct computation, one obtains

$$
\operatorname{div}\left(\sigma \nabla S_{n}\right)=\sigma(2 i \eta+1 / n) r^{i \eta-2+1 / n} \varphi(\theta) / n .
$$

\footnotetext{
${ }^{3}$ More precisely, one finds $\left.\varphi\right|_{\Omega_{1}}=a_{1} \sinh (\eta \theta)+b_{1} \cosh (\eta \theta)$ and $\left.\varphi\right|_{\Omega_{2}}=a_{2} \sinh (\eta \theta)+b_{2} \cosh (\eta \theta)$, where the constants $a_{1}, a_{2}$, $b_{1}, b_{2}$ are chosen to ensure matching traces and fluxes on the interface $\Sigma$.
} 
Integrating by parts with respect to the variable $r$, one can write

$$
\begin{aligned}
-\left(\operatorname{div}\left(\sigma \nabla S_{n}\right), \chi v\right)_{\Omega} & =-(1 / n) \int_{0}^{2 \pi} \int_{0}^{d} \sigma(2 i \eta+1 / n) r^{i \eta-2+1 / n} \varphi(\theta)(\overline{\chi v}) r \mathrm{~d} r \mathrm{~d} \theta \\
& =(1 / n) \int_{0}^{2 \pi} \int_{0}^{d} \sigma(2 i \eta+1 / n) \frac{r^{i \eta-1+1 / n}}{i \eta+1 / n} \varphi(\theta) \frac{\partial(\overline{\chi v})}{\partial r} r \mathrm{~d} r \mathrm{~d} \theta .
\end{aligned}
$$

Cauchy-Schwarz inequality leads to

$$
\left|\left(\sigma \nabla S_{n}, \nabla(\chi v)\right)_{\Omega}\right| \leq(C / n)\left(\int_{0}^{2 \pi} \int_{0}^{d}|\sigma(2 i \eta+1 / n)|^{2} \frac{r^{-2+2 / n}}{|i \eta+1 / n|^{2}}|\varphi(\theta)|^{2} r \mathrm{~d} r \mathrm{~d} \theta\right)^{1 / 2}\|v\|_{H_{0}^{1}(\Omega)} .
$$

But,

$$
(1 / n)^{2} \int_{0}^{2 \pi} \int_{0}^{d}|\sigma(2 i \eta+1 / n)|^{2} \frac{r^{-2+2 / n}}{|i \eta+1 / n|^{2}}|\varphi(\theta)|^{2} r \mathrm{~d} r \mathrm{~d} \theta \leq C(1 / n)^{2} \int_{0}^{d} r^{-2+2 / n} r \mathrm{~d} r \leq C / n .
$$

Thus,

$$
\left|\left(\sigma \nabla S_{n}, \nabla(\chi v)\right)_{\Omega}\right| \leq C\|v\|_{H_{0}^{1}(\Omega)} / \sqrt{n} .
$$

Plugging (6.11) and (6.12) in (6.10), one finally finds

$$
\left\|\operatorname{div}\left(\sigma \nabla u_{n}\right)\right\|_{H^{-1}(\Omega)} \leq C\left(\left\|S_{n}\right\|_{\Omega}+1 / \sqrt{n}\right) .
$$

Now, we recall that $\left(S_{n}\right)_{n \in \mathbb{N}^{*}}$ is bounded in $L^{2}(\Omega)$. As a consequence, the limit (6.9) and Inequality (6.13), together with Lemma 4.5 , prove that $A$ is not a Fredholm operator in the case where $\left.\kappa_{\sigma} \in\right]-I_{\alpha^{i}} ;-1 / I_{\alpha^{i}}[\backslash\{-1\}$.

The cases 2 . and 3 . of Theorem 6.4 can be treated in a similar way.

\subsection{Further comments}

Let us conclude by two cases not covered by Theorem 4.3 .

First, a domain $\Omega:=]-1 ; 1[\times]-1 ; 1\left[\right.$, with subsets $\left.\Omega_{1}:=\right]-1 ; 0[\times]-1 ; 1\left[\right.$ and $\left.\Omega_{2}:=\right] 0 ; 1[\times]-1 ; 1[$ (see Fig. 11-left). Assume that $\sigma=1$ in $\Omega_{1}, \sigma=-2$ in $] 0 ; 1[\times] 0 ; 1\left[\right.$ in $\sigma=\beta \in \mathbb{R}_{-}^{*}$ in $] 0 ; 1[\times]-1 ; 0[$. Given $\beta>-1$, there holds, for all $d>0$,

$$
\inf _{B(O, d) \cap \Omega_{1}} \sigma_{1}<\sup _{B(O, d) \cap \Omega_{2}}\left|\sigma_{2}\right| \text { and } \inf _{B(O, d) \cap \Omega_{2}}\left|\sigma_{2}\right|<\sup _{B(O, d) \cap \Omega_{1}} \sigma_{1} .
$$

So, the assumptions of Theorem 4.3 are not fulfilled and, as a consequence, one can not conclude that the operator $A$ of $\mathcal{L}\left(H_{0}^{1}(\Omega), H^{-1}(\Omega)\right)$ is Fredholm.

Remark 6.6. However, one can easily build by hand, for this simple configuration, an ad hoc operator $T$ that allows one to prove $T$-coercivity directly for some $\beta>-1$. For that, the operator $T$ is built using some line symmetries. For $u \in H_{0}^{1}(\Omega)$, the action of $T$ is defined by

$$
(T u)(x, y):= \begin{cases}u_{a}(x, y)-2 u_{d}(-x, y) & \text { in } \left.\Omega_{a}:=\right]-1 ; 0[\times] 0 ; 1[ \\ u_{b}(x, y)-2 u_{d}(-x,-y) & \text { in } \left.\Omega_{b}:=\right]-1 ; 0[2 \\ -2 u_{a}(-x,-y)+2 u_{b}(-x, y)-u_{c}(x, y) & \text { in } \left.\Omega_{c}:=\right] 0 ; 1[\times]-1 ; 0[ \\ -u_{d}(x, y) & \text { in } \left.\Omega_{d}:=\right] 0 ; 1\left[{ }^{2}\right.\end{cases}
$$

with $u_{k}:=\left.u\right|_{\Omega_{k}}$, for $k=a, b, c, d$. 

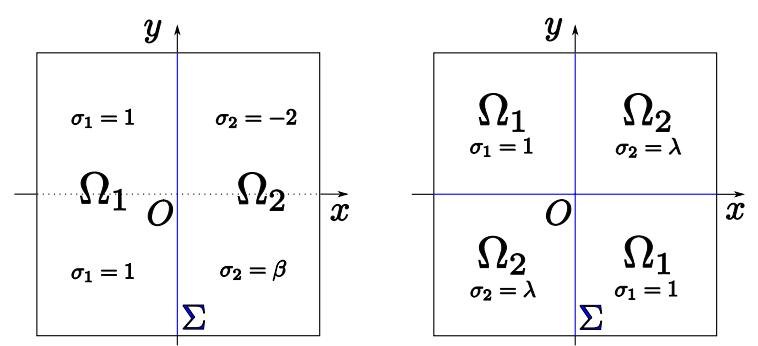

Figure 11. Two situations not covered by Theorem 4.3: $\beta>-1$ on the left; $\lambda \in \mathbb{R}_{-}^{*}$ on the right.

On the other hand, if $\beta<-1$, then

$$
\inf _{B(O, d) \cap \Omega_{2}}\left|\sigma_{2}\right|>\sup _{B(O, d) \cap \Omega_{1}} \sigma_{1}
$$

and Theorem 4.3 allows one to conclude that $A$ is Fredholm (of index 0 ).

Second, a domain $\Omega:=]-1 ; 1[\times]-1 ; 1\left[\right.$, with subsets $\left.\Omega_{1}:=\right]-1 ; 0[\times] 0 ; 1[\cup] 0 ; 1[\times]-1 ; 0\left[\right.$ and $\Omega_{2}:=$ ]$-1 ; 0[\times]-1 ; 0[\cup] 0 ; 1[\times] 0 ; 1[$ (see Fig. 11-right). Here, one cannot use Theorem 4.3, because the boundaries $\partial \Omega_{1}$ and $\partial \Omega_{2}$ are not Lipschitz (see [3], Corrigendum).

\section{Domains of $\mathbb{R}^{3}$}

Generally speaking, one can use the same lines of thought to tackle the problem $(\mathscr{P})$ in a domain $\Omega$ of $\mathbb{R}^{3}$. Provided one can establish $T$-coercivity locally (cf. Sect. 3 ), one can prove that the operator $A \in \mathcal{L}\left(H_{0}^{1}(\Omega), H^{-1}(\Omega)\right)$ is Fredholm. The main difference is that one has to deal with a larger number of elementary cases, and among them some can not be reduced to their lower-dimensional counterparts. Notations used previously are kept here.

We begin the study by elementary cases. We provide proofs only in the most illustrative cases.

\subsection{Symmetric domain of $\mathbb{R}^{3}$}

One obtains easily the same results as the ones stated in Theorem 3.1.

\subsection{Prismatic edges}

Introduce the cylindrical coordinates $(r, \theta, z)$ centered on the edge, so that the cartesian coordinates are mapped as $(x, y, z)=(r \cos \theta, r \sin \theta, z)$. Let $H>0$ denote the height of the cylinder, $R>0$ its radius.

\subsubsection{Interior edge}

Consider the geometry of Figure 12-left. Given $0<\alpha<2 \pi$, define

$$
\begin{aligned}
& \Omega_{1}:=\{(r \cos \theta, r \sin \theta, z) \mid 0<r<R, 0<\theta<\alpha, 0<z<H\} ; \\
& \Omega_{2}:=\{(r \cos \theta, r \sin \theta, z) \mid 0<r<R, \alpha<\theta<2 \pi, 0<z<H\} .
\end{aligned}
$$

Theorem 7.1 (interior edge in 3D). Assume that

$$
\max \left(\sigma_{1}^{-} / \sigma_{2}^{+}, \sigma_{2}^{-} / \sigma_{1}^{+}\right)>I_{\alpha}, \quad \text { with } \quad I_{\alpha}:=\max \left(\frac{\alpha}{2 \pi-\alpha}, \frac{2 \pi-\alpha}{\alpha}\right) .
$$

Then, there exists an isomorphism $T \in \mathcal{L}\left(H_{0}^{1}(\Omega)\right)$ such that the form a is $T$-coercive and $A: u \mapsto-\operatorname{div}(\sigma \nabla u)$ is an isomorphism from $H_{0}^{1}(\Omega)$ to $H^{-1}(\Omega)$. 

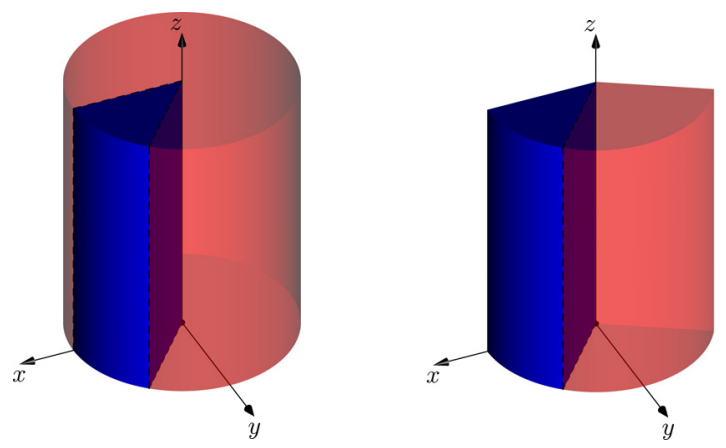

FIGURE 12. Geometry of prismatic edges: (left) interior edge. (right) Boundary edge.

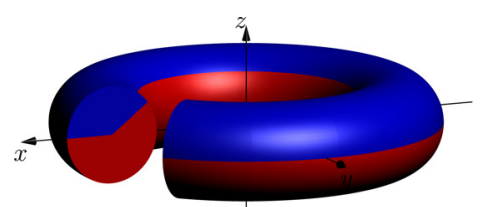

FIGURE 13. Geometry of an interior axisymmetric edge.

Proof. Define the two operators $R_{1}$ and $R_{2}$ respectively by $\left(R_{1} u_{1}\right)(\rho, \Theta, Z)=u_{1}\left(\rho, \frac{\alpha}{\alpha-2 \pi}(\Theta-2 \pi), Z\right)$ and $\left(R_{2} u_{2}\right)(\rho, \Theta, Z)=u_{2}\left(\rho, \frac{\alpha-2 \pi}{\alpha} \Theta+2 \pi, Z\right)$. As before, the matching condition holds for $R_{1}$. We find as in Theorem 3.3 that $\left\|R_{1}\right\|^{2} \leq I_{\alpha}$. Similarly, the matching condition holds for $R_{2}$ and $\left\|R_{2}\right\|^{2} \leq I_{\alpha}$. We conclude the proof as usual (see Thm. 2.1).

\subsubsection{Boundary edge}

Consider the geometry of Figure 12-right. Given $0<\alpha<\gamma<2 \pi$, define

$$
\begin{aligned}
& \Omega_{1}:=\{(r \cos \theta, r \sin \theta, z) \mid 0<r<R, 0<\theta<\alpha, 0<z<H\} ; \\
& \Omega_{2}:=\{(r \cos \theta, r \sin \theta, z) \mid 0<r<R, \alpha<\theta<\gamma, 0<z<H\} .
\end{aligned}
$$

One obtains the same results as the ones of Theorem 3.7.

\subsection{Axisymmetric edges}

We refer to the geometry of Figure 13, with toroidal coordinates $(r, \theta, \varphi)$ such that cartesian coordinates are mapped as $(x, y, z)=(\cos \theta(R+r \cos \varphi), \sin \theta(R+r \cos \varphi), r \sin \varphi)$. Here, $R>0$ denotes the radius of the torus. Given $0<d<R$ and $0<\alpha<2 \pi$, define

$$
\begin{aligned}
& \Omega_{1}:=\{(\cos \theta(R+r \cos \varphi), \sin \theta(R+r \cos \varphi), r \sin \varphi) \mid 0<r<d, 0 \leq \theta<2 \pi, 0<\varphi<\alpha\} ; \\
& \Omega_{2}:=\{(\cos \theta(R+r \cos \varphi), \sin \theta(R+r \cos \varphi), r \sin \varphi) \mid 0<r<d, 0 \leq \theta<2 \pi, \alpha<\varphi<2 \pi\} .
\end{aligned}
$$

Theorem 7.2 (axisymmetric interior edge in 3D). Assume that

$$
\max \left(\sigma_{1}^{-} / \sigma_{2}^{+}, \sigma_{2}^{-} / \sigma_{1}^{+}\right)>\frac{1+d / R}{1-d / R} I_{\alpha}, \quad \text { with } \quad I_{\alpha}:=\max \left(\frac{\alpha}{2 \pi-\alpha}, \frac{2 \pi-\alpha}{\alpha}\right) .
$$

Then, there exists an isomorphism $T \in \mathcal{L}\left(H_{0}^{1}(\Omega)\right)$ such that the form a is $T$-coercive and $A: u \mapsto-\operatorname{div}(\sigma \nabla u)$ is an isomorphism from $H_{0}^{1}(\Omega)$ to $H^{-1}(\Omega)$. 


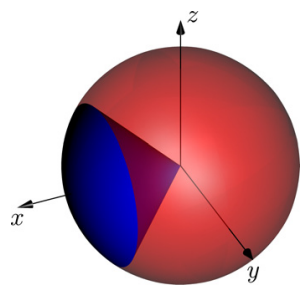

FiguRE 14. Geometry of an interior conical vertex.

Proof. Introduce the operators $R_{1}$ and $R_{2}$ respectively defined by $\left(R_{1} u_{1}\right)(\rho, \Theta, \Phi)=u_{1}\left(\rho, \Theta, \frac{\alpha}{\alpha-2 \pi}(\Phi-2 \pi)\right)$ and $\left(R_{2} u_{2}\right)(\rho, \Theta, \Phi)=u_{2}\left(\rho, \Theta, \frac{\alpha-2 \pi}{\alpha} \Phi+2 \pi\right)$. The matching conditions hold for $R_{1}$ and $R_{2}$.

To compute the norm of $R_{1}$, let $u_{1} \in H_{0, \Gamma_{1}}^{1}\left(\Omega_{1}\right)$. With the help of the change of (toroidal) variables $(r, \theta, \varphi)=$ $\left(\rho, \Theta, \frac{\alpha}{\alpha-2 \pi}(\Phi-2 \pi)\right)$, we find $^{4}$

$$
\begin{aligned}
\left\|\nabla\left(R_{1} u_{1}\right)\right\|_{\Omega_{2}}^{2}= & \int_{\Omega_{2}}\left(\left(\frac{\partial\left(R_{1} u_{1}\right)}{\partial \rho}\right)^{2}+\frac{1}{(R+\rho \cos \Phi)^{2}}\left(\frac{\partial\left(R_{1} u_{1}\right)}{\partial \Theta}\right)^{2}\right) \rho(R+\rho \cos \Phi) \mathrm{d} \rho \mathrm{d} \Phi \mathrm{d} \Theta \\
& +\int_{\Omega_{2}} \frac{1}{\rho^{2}}\left(\frac{\partial\left(R_{1} u_{1}\right)}{\partial \Phi}\right)^{2} \rho(R+\rho \cos \Phi) \mathrm{d} \rho \mathrm{d} \Phi \mathrm{d} \Theta \\
\leq & \frac{2 \pi-\alpha}{\alpha} \int_{\Omega_{1}}\left(\frac{\partial u_{1}}{\partial r}\right)^{2} r\left(R+r \cos \left(\frac{2 \pi-\alpha}{\alpha} \varphi\right)\right) \mathrm{d} r \mathrm{~d} \varphi \mathrm{d} \theta \\
& +\frac{2 \pi-\alpha}{\alpha} \int_{\Omega_{1}} \frac{1}{\left(R+r \cos \left(\frac{2 \pi-\alpha}{\alpha} \varphi\right)\right)^{2}}\left(\frac{\partial u_{1}}{\partial \theta}\right)^{2} r\left(R+r \cos \left(\frac{2 \pi-\alpha}{\alpha} \varphi\right)\right) \mathrm{d} r \mathrm{~d} \varphi \mathrm{d} \theta \\
& +\frac{\alpha}{2 \pi-\alpha} \int_{\Omega_{1}} \frac{1}{r^{2}}\left(\frac{\partial u_{1}}{\partial \varphi}\right)^{2} r\left(R+r \cos \left(\frac{2 \pi-\alpha}{\alpha} \varphi\right)\right) \mathrm{d} r \mathrm{~d} \varphi \mathrm{d} \theta .
\end{aligned}
$$

By direct inspection, one finds

$$
\left.\frac{R+r \cos \left(\frac{2 \pi-\alpha}{\alpha} \theta\right)}{R+r \cos \theta} \leq \frac{1+d / R}{1-d / R} \quad \text { and } \quad \frac{R+r \cos \theta}{R+r \cos \left(\frac{2 \pi-\alpha}{\alpha} \theta\right)} \leq \frac{1+d / R}{1-d / R}, \quad \forall r \in\right] 0 ; d[, \quad \forall \theta \in] 0 ; \alpha[,
$$

so one obtains $\left\|R_{1}\right\|^{2} \leq \frac{1+d / R}{1-d / R} I_{\alpha}$. Similarly, $\left\|R_{2}\right\|^{2} \leq \frac{1+d / R}{1-d / R} I_{\alpha}$. We conclude as in the proof of Theorem 2.1.

Remark 7.3. If $\max \left(\sigma_{1}^{-} / \sigma_{2}^{+}, \sigma_{2}^{-} / \sigma_{1}^{+}\right)>I_{\alpha}$, then according to Theorem $7.2, A: u \mapsto-\operatorname{div}(\sigma \nabla u)$ is an isomorphism from $H_{0}^{1}(\Omega)$ to $H^{-1}(\Omega)$ for $d / R$ small enough.

Remark 7.4. We focused here on the case of an interior axisymmetric edge. Boundary axisymmetric edges can be handled as before, with a final result like Theorem 3.7.

\subsection{Conical vertex}

Consider the geometry of Figure 14, and the associated spherical coordinates $(r, \theta, \varphi)$ centered at the origin. The cartesian coordinates are now mapped as $(x, y, z)=(r \cos \theta, r \sin \theta \cos \varphi, r \sin \theta \sin \varphi)$. Let $R>0$ and $0<\alpha<\pi$, and define

$$
\begin{aligned}
& \Omega_{1}:=\{(r \cos \theta, r \sin \theta \cos \varphi, r \sin \theta \sin \varphi), 0<r<R, 0 \leq \theta<\alpha, 0 \leq \varphi<2 \pi\} ; \\
& \Omega_{2}:=\{(r \cos \theta, r \sin \theta \cos \varphi, r \sin \theta \sin \varphi), 0<r<R, \alpha<\theta \leq \pi, 0 \leq \varphi<2 \pi\} .
\end{aligned}
$$

\footnotetext{
${ }^{4}$ See Section 8.3 for complementary computations.
} 
Theorem 7.5 (conical interior vertex in 3D). Assume that ${ }^{5}$

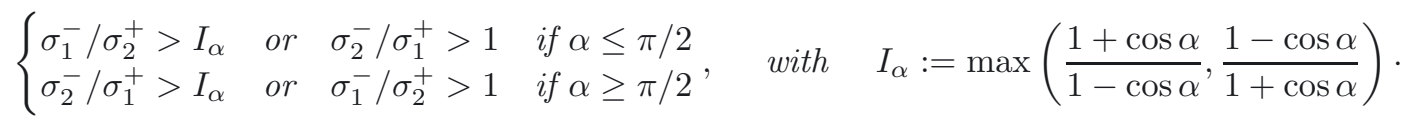

Then, there exists an isomorphism $T \in \mathcal{L}\left(H_{0}^{1}(\Omega)\right)$ such that the form a is $T$-coercive and $A: u \mapsto-\operatorname{div}(\sigma \nabla u)$ is an isomorphism from $H_{0}^{1}(\Omega)$ to $H^{-1}(\Omega)$.

Proof. We consider the case $\alpha \leq \pi / 2$.

Define the operators $R_{1}$ and $R_{2}$ by $\left(R_{1} u_{1}\right)(\rho, \Theta, \Phi)=u_{1}\left(\rho, g_{1}(\Theta), \Phi\right)$, and by $\left(R_{2} u_{2}\right)(\rho, \Theta, \Phi)=$ $u_{2}\left(\rho, g_{2}(\Theta), \Phi\right)$. Here, $g_{1}$ is a $\mathscr{C}^{1}$ diffeomorphism from $[\alpha ; \pi]$ to $[0 ; \alpha]$ such that $g_{1}(\pi)=0$ and $g_{1}(\alpha)=\alpha$ whereas $g_{2}$ is a $\mathscr{C}^{1}$ diffeomorphism from $[0 ; \alpha]$ to $[\alpha ; \pi]$ such that $g_{2}(0)=\pi$ and $g_{2}(\alpha)=\alpha$. We denote $h_{1}$ (resp. $h_{2}$ ) the inverse of $g_{1}$ (resp. $\left.g_{2}\right)$.

The matching conditions hold. We evaluate the norm of $R_{1}$. Let $u_{1} \in H_{0, \Gamma_{1}}^{1}\left(\Omega_{1}\right)$. Performing the change of variables $(r, \theta, \varphi)=\left(\rho, g_{1}(\Theta), \Phi\right)$, we find successively

$$
\begin{aligned}
\left\|\nabla\left(R_{1} u_{1}\right)\right\|_{\Omega_{2}}^{2}= & \int_{\Omega_{2}}\left(\frac{\partial\left(R_{1} u_{1}\right)}{\partial \rho}\right)^{2}+\frac{1}{\rho^{2}}\left(\frac{\partial\left(R_{1} u_{1}\right)}{\partial \Theta}\right)^{2}+\frac{1}{(\rho \sin \Theta)^{2}}\left(\frac{\partial\left(R_{1} u_{1}\right)}{\partial \Phi}\right)^{2} \rho^{2} \mathrm{~d} \rho \sin \Theta \mathrm{d} \Theta \mathrm{d} \Phi \\
\leq & \int_{\Omega_{1}}\left(\frac{\partial u_{1}}{\partial r}\right)^{2} r^{2} \mathrm{~d} r \sin \left(h_{1}(\theta)\right)\left|h_{1}^{\prime}(\theta)\right| \mathrm{d} \theta \mathrm{d} \varphi \\
& +\int_{\Omega_{1}} \frac{1}{r^{2}\left|h_{1}^{\prime}(\theta)\right|^{2}}\left(\frac{\partial u_{1}}{\partial \theta}\right)^{2} r^{2} \mathrm{~d} r \sin \left(h_{1}(\theta)\right)\left|h_{1}^{\prime}(\theta)\right| \mathrm{d} \theta \mathrm{d} \varphi \\
& +\int_{\Omega_{1}} \frac{1}{\left(r \sin h_{1}(\theta)\right)^{2}}\left(\frac{\partial u_{1}}{\partial \varphi}\right)^{2} r^{2} \mathrm{~d} r \sin \left(h_{1}(\theta)\right)\left|h_{1}^{\prime}(\theta)\right| \mathrm{d} \theta \mathrm{d} \varphi .
\end{aligned}
$$

We get the bound

$$
\left\|R_{1}\right\|^{2} \leq \max \left(\left\|\frac{\left|h_{1}^{\prime}(\theta)\right| \sin \left(h_{1}(\theta)\right)}{\sin \theta}\right\|_{L^{\infty}(] 0 ; \alpha[)},\left\|\frac{\sin \left(h_{1}(\theta)\right)}{\left|h_{1}^{\prime}(\theta)\right| \sin \theta}\right\|_{L^{\infty}(] 0 ; \alpha[)},\left\|\frac{\left|h_{1}^{\prime}(\theta)\right| \sin \theta}{\sin \left(h_{1}(\theta)\right)}\right\|_{L^{\infty}(] 0 ; \alpha[)}\right) .
$$

Our aim is to exhibit an explicit admissible function $\theta \mapsto h_{1}(\theta)$ which yields a right-hand side, as small as possible. To achieve this end, we consider the following strategy: use functions $h_{1}$ such that one of the three above quotients is constant with respect to $\theta$. More to the point, we take the map

$$
\left.h_{1}(\theta)=\arccos \left(\frac{\cos \alpha+1}{\cos \alpha-1} \cos \theta-2 \frac{\cos \alpha}{\cos \alpha-1}\right), \quad \text { such that } \frac{\left|h_{1}^{\prime}(\theta)\right| \sin \left(h_{1}(\theta)\right)}{\sin \theta}=\frac{1+\cos \alpha}{1-\cos \alpha}, \quad \forall \theta \in\right] 0 ; \alpha[.
$$

One finds also

$$
\left.\frac{\sin \left(h_{1}(\theta)\right)}{\left|h_{1}^{\prime}(\theta)\right| \sin \theta} \leq 1, \quad \frac{\left|h_{1}^{\prime}(\theta)\right| \sin \theta}{\sin \left(h_{1}(\theta)\right)} \leq \frac{1+\cos \alpha}{1-\cos \alpha}, \quad \forall \theta \in\right] 0 ; \alpha[.
$$

Thus, there holds $\left\|R_{1}\right\|^{2} \leq I_{\alpha}$.

On the other hand, for $R_{2}$, we have to minimize

$$
\max \left(\left\|\frac{\left|h_{2}^{\prime}(\theta)\right| \sin \left(h_{2}(\theta)\right)}{\sin \theta}\right\|_{L^{\infty}(] \alpha ; \pi[)},\left\|\frac{\sin \left(h_{2}(\theta)\right)}{\left|h_{2}^{\prime}(\theta)\right| \sin \theta}\right\|_{L^{\infty}(] \alpha ; \pi[)},\left\|\frac{\left|h_{2}^{\prime}(\theta)\right| \sin \theta}{\sin \left(h_{2}(\theta)\right)}\right\|_{L^{\infty}(] \alpha ; \pi[)}\right) .
$$

Let us consider the stereographic map

$$
g_{2}(\Theta)=2 \arctan \left(\frac{\tan (\alpha / 2)^{2}}{\tan (\Theta / 2)}\right) \quad \text { so } \quad h_{2}(\theta)=2 \arctan \left(\frac{\tan (\alpha / 2)^{2}}{\tan (\theta / 2)}\right) .
$$

\footnotetext{
${ }^{5}$ The ratio $(1+\cos \alpha) /(1-\cos \alpha)$ is equal to the ratio of the solid angles.
} 
One finds

$$
\left.\frac{\left|h_{2}^{\prime}(\theta)\right| \sin \left(h_{2}(\theta)\right)}{\sin \theta} \leq 1, \quad \frac{\sin \left(h_{2}(\theta)\right)}{\left|h_{2}^{\prime}(\theta)\right| \sin \theta}=1, \quad \frac{\left|h_{2}^{\prime}(\theta)\right| \sin \theta}{\sin \left(h_{2}(\theta)\right)}=1, \quad \forall \theta \in\right] \alpha ; \pi[,
$$

so $\left\|R_{2}\right\|^{2} \leq 1$. We conclude as in the proof of Theorem 2.1 .

One proceeds similarly to deal with the case $\pi / 2<\alpha<\pi$.

Remark 7.6. In the case of the conical vertex, it is an open question to prove that the interval obtained in Theorem 7.5, with this particular choice of $R_{1}$ and $R_{2}$, is optimal. In other words, when the contrast lies in the interval, which is, surprisingly, not "symmetric" with respect to -1 , we do not know whether or not the operator $A$ is Fredholm. To address this question, we would have to compute the singularities but the computations are much more involved than in a $2 \mathrm{D}$ configuration.

\subsection{Fichera's corner}

In a domain of $\mathbb{R}^{3}$, it can happen that edges and vertices interact with one another, in ways which are not covered by the approach we developed before for domains of $\mathbb{R}^{2}$. To illustrate this situation, we consider a wellknown example, the so-called Fichera's corner. More precisely, let us define $\Omega:=]-1 ; 1\left[^{3}\right.$, with $\left.\Omega_{1}:=\right] 0 ; 1\left[^{3}\right.$, and $\Omega_{2}$ the open set such that $\Omega_{2}:=\Omega \backslash \overline{\Omega_{1}}$.

Theorem 7.7 (Fichera's corner). Assume that

$$
\max \left(\sigma_{1}^{-} / \sigma_{2}^{+}, \sigma_{2}^{-} / \sigma_{1}^{+}\right)>7 .
$$

Then, there exists an isomorphism $T \in \mathcal{L}\left(H_{0}^{1}(\Omega)\right)$ such that the form a is $T$-coercive and $A: u \mapsto-\operatorname{div}(\sigma \nabla u)$ is an isomorphism from $H_{0}^{1}(\Omega)$ to $H^{-1}(\Omega)$.

Proof. With the help of reflection symmetries ${ }^{6}$, we define the operator $R_{1}$ by

$$
\left(R_{1} u_{1}\right)(x, y, z)= \begin{cases}u_{1}(-x, y, z) & \text { in } \left.\Omega_{2}^{1}:=\right]-1 ; 0[\times] 0 ; 1\left[^{2}\right. \\ u_{1}(x,-y, z) & \text { in } \left.\Omega_{2}^{2}:=\right] 0 ; 1[\times]-1 ; 0[\times] 0 ; 1[ \\ u_{1}(x, y,-z) & \text { in } \left.\Omega_{2}^{3}:=\right] 0 ; 1\left[^{2} \times\right]-1 ; 0[ \\ u_{1}(-x,-y, z) & \text { in } \left.\Omega_{2}^{4}:=\right]-1 ; 0\left[^{2} \times\right] 0 ; 1[ \\ u_{1}(-x, y,-z) & \text { in } \left.\Omega_{2}^{5}:=\right]-1 ; 0[\times] 0 ; 1[\times]-1 ; 0[ \\ u_{1}(x,-y,-z) & \text { in } \left.\Omega_{2}^{6}:=\right] 0 ; 1[\times]-1 ; 0\left[^{2}\right. \\ u_{1}(-x,-y,-z) & \text { in } \left.\Omega_{2}^{7}:=\right]-1 ; 0\left[^{3} .\right.\end{cases}
$$

Next, we define $R_{2}$ by

$$
\begin{aligned}
\left(R_{2} u_{2}\right)(x, y, z)= & u_{2}^{1}(-x, y, z)+u_{2}^{2}(x,-y, z)+u_{2}^{3}(x, y,-z)-u_{2}^{4}(-x,-y, z) \\
& -u_{2}^{5}(-x, y,-z)-u_{2}^{6}(x,-y,-z)+u_{2}^{7}(-x,-y,-z) .
\end{aligned}
$$

Above, $\left(u_{2}^{\ell}\right)_{\ell=1,7}$ respectively denote the restriction of $u_{2}$ to $\left(\Omega_{2}^{\ell}\right)_{\ell=1,7}$.

The matching conditions hold. Then, one obtains easily that for all $u_{1} \in H_{0, \Gamma_{1}}^{1}\left(\Omega_{1}\right),\left\|\nabla\left(R_{1} u_{1}\right)\right\|_{\Omega_{2}}^{2}=$ $7\left\|\nabla u_{1}\right\|_{\Omega_{1}}^{2}$. On the other hand, for all $u_{2} \in H_{0, \Gamma_{2}}^{1}\left(\Omega_{2}\right),\left\|\nabla\left(R_{2} u_{2}\right)\right\|_{\Omega_{1}}^{2} \leq 7\left\|\nabla u_{2}\right\|_{\Omega_{2}}^{2}$. Indeed, there holds classically $\left(\sum_{k=1}^{7} a_{k}\right)^{2} \leq 7 \sum_{k=1}^{7} a_{k}^{2}$, for all $\left(a_{1}, \ldots, a_{7}\right) \in \mathbb{R}^{7}$. We conclude as in the proof of Theorem 2.1.

\footnotetext{
${ }^{6} \mathrm{~A}$ similar approach has been recently used by Nicaise and Venel in [18] in a geometry of $\mathbb{R}^{2}$, with $\left.\Omega:=\right]-1 ; 1\left[{ }^{2}\right.$ and $\left.\Omega_{1}:=\right] 0 ; 1\left[{ }^{2}\right.$.
} 


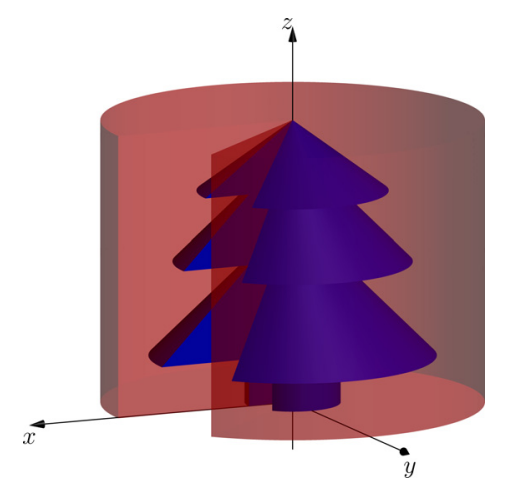

Figure 15. Joyeux Noël, aka. Merry Christmas!

\subsection{General geometries in $\mathbb{R}^{3}$}

To establish that the operator $A$ is Fredholm, in the case of general geometries in $\mathbb{R}^{3}$ (see for instance Fig. 15), one can proceed by localization, as in Section 4 (cf. Thm. 4.3) and Section 5. Also, one can prove optimality results, in the same spirit of Section 6 . We do not provide the details here, but instead comment on the case of Fichera's corner. For simplicity, let us consider constant coefficients $\sigma_{1}$ and $\sigma_{2}$, and a situation in which the contrast $\kappa_{\sigma}=\sigma_{2} / \sigma_{1}$ lies within the critical interval $[-7 ;-1 / 7]$, i.e. the case not covered by Theorem 7.7 . Loosely speaking, one finds that

- If $\kappa_{\sigma}=-1$ then there exists a surface singularity distribution. Indeed, at each point standing on one of the three (open) faces of the interface, one can build a sequence of functions that prevents $A$ from being Fredholm. To achieve this result, one extends the construction given in the proof of Theorem 6.2.

- If $\left.\kappa_{\sigma} \in\right]-3 ;-1 / 3[$ then there exists in addition a linear singularity distribution: at each point standing on one of the three (open) lines of the interface, one can build a sequence of functions that prevents $A$ from being Fredholm, using the pointwise singularities exhibited in Theorem 6.4.

- If $\left.\kappa_{\sigma} \in\right]-7 ;-1 / 7[$ then there exists in addition a pointwise singularity, which can be build in the same spirit as those of Theorem 6.4.

\section{Missing COMPUTATIONS}

\subsection{Computations for Theorem 6.2}

Let $b>0$ such that $[-b ; b] \times[-b ; b] \subset \Omega$. We define $\left(u_{n}\right)_{n}$ as in $(6.2)$, and a cutoff function $\chi \in \mathscr{C}_{0}^{\infty}\left(\mathbb{R}^{2}\right)$, equal to 1 in $[-b / 2 ; b / 2] \times[-b / 2 ; b / 2]$.

Lemma 8.1. There holds $\left\|\chi u_{n}\right\|_{H_{0}^{1}(\Omega)} \underset{n \rightarrow+\infty}{\longrightarrow}+\infty$.

Proof. Introduce $\tilde{D}:=[-b / 2 ; b / 2] \times[-b / 2 ; b / 2]$, and write

$$
\begin{aligned}
\left\|\chi u_{n}\right\|_{H_{0}^{1}(\Omega)}^{2} & \geq\left\|\nabla u_{n}\right\|_{\tilde{D}}^{2} \geq\left\|\partial_{t} u_{n}\right\|_{\tilde{D}}^{2} \\
& \geq 2 \int_{-b / 2}^{b / 2} \int_{0}^{b / 2} n^{2} \cos ^{2} n t \frac{\sinh ^{2} n(b-s)}{\mathrm{e}^{2 n b}} \mathrm{~d} s \mathrm{~d} t \\
& \geq 2 n^{2} \int_{-b / 2}^{b / 2} \cos ^{2} n t \mathrm{~d} t \int_{0}^{b / 2} \frac{\sinh ^{2} n(b-s)}{\mathrm{e}^{2 n b}} \mathrm{~d} s \\
& \geq 2 n^{2}\left[\frac{b}{2}+\frac{\sin n b}{2 n}\right] \int_{0}^{b / 2} \frac{\sinh ^{2} n(b-s)}{\mathrm{e}^{2 n b}} \mathrm{~d} s .
\end{aligned}
$$


But one has

$$
\begin{aligned}
4 \int_{0}^{b / 2} \frac{\sinh ^{2} n(b-s)}{\mathrm{e}^{2 n b} \mathrm{~d} s} & =\int_{0}^{b / 2} \mathrm{e}^{-2 n s}-2 \mathrm{e}^{-2 n b}+\mathrm{e}^{2 n s-4 n b} \mathrm{~d} s \\
& =\left(\frac{1}{2 n}-\frac{\mathrm{e}^{-n b}}{2 n}\right)-\left(b \mathrm{e}^{-2 n b}\right)+\mathrm{e}^{-4 n b}\left(\frac{\mathrm{e}^{n b}}{2 n}-\frac{1}{2 n}\right) \sim \frac{1}{2 n}
\end{aligned}
$$

Hence, there exists $C>0$, such that for large $n$, one has $\left\|\chi u_{n}\right\|_{H_{0}^{1}(\Omega)}^{2}>C n$.

\subsection{Computations for Theorem 6.4}

Define $u_{n}(r, \theta):=\chi(r) S_{n}(r, \theta)$ where $\chi$ is a cut-off function equal to 1 for $0 \leq r \leq d / 2$ and $S_{n}(r, \theta):=$ $r^{i \eta+1 / n} \varphi(\theta)$.

Lemma 8.2. There holds $\left\|u_{n}\right\|_{H_{0}^{1}(\Omega)} \underset{n \rightarrow+\infty}{\longrightarrow}+\infty$.

Proof. One writes

$$
\begin{aligned}
\left\|u_{n}\right\|_{H_{0}^{1}(\Omega)}^{2} & \geq \int_{0}^{2 \pi} \int_{0}^{d / 2} r^{-2+2 / n}\left|\partial_{\theta} \varphi\right|^{2} r \mathrm{~d} r \mathrm{~d} \theta \\
& \geq C \int_{0}^{d / 2} r^{-1+2 / n} \mathrm{~d} r \\
& \geq C n(d / 2)^{2 / n} / 2 \underset{n \rightarrow \infty}{\longrightarrow}+\infty .
\end{aligned}
$$

\subsection{Toroidal coordinates}

Considering the geometry of Figure 13, introduce the change of variables $(x, y, z)=(\cos \theta(R+$ $r \cos \varphi), \sin \theta(R+r \cos \varphi), r \sin \varphi)$, for $R>0$. The jacobian associated with this change of variables is

$$
\left(\begin{array}{ccc}
\cos \theta \cos \varphi & -\sin \theta(R+r \cos \varphi) & -r \cos \theta \sin \varphi \\
\sin \theta \cos \varphi & \cos \theta(R+r \cos \varphi) & -r \sin \theta \sin \varphi \\
\sin \varphi & 0 & r \cos \varphi
\end{array}\right) .
$$

The elementary volume in toroidal coordinates is then $r(R+r \cos \varphi) \mathrm{d} r \mathrm{~d} \varphi \mathrm{d} \theta$.

Also, the gradient in toroidal coordinates writes

$$
\nabla u=\left(\begin{array}{c}
\frac{\partial u}{\partial r} \\
\frac{1}{R+r \cos \varphi} \frac{\partial u}{\partial \theta} \\
\frac{1}{r} \frac{\partial u}{\partial \varphi}
\end{array}\right)
$$

Acknowledgements. The authors thank Monique Dauge for pointing out the possible use of the singularities $S_{n}(r, \theta)$ in the proof of Theorem 6.4 and Xavier Claeys for suggesting the use of the map $g_{2}$ in the proof of Theorem 7.5.

\section{REFERENCES}

[1] A.-S. Bonnet-Ben Dhia, L. Chesnel and X. Claeys, Radiation condition for a non-smooth interface between a dielectric and a metamaterial [hal-00651008].

[2] A.-S. Bonnet-Ben Dhia, P. Ciarlet Jr. and C.M. Zwölf, A new compactness result for electromagnetic waves. Application to the transmission problem between dielectrics and metamaterials. Math. Models Methods Appl. Sci. 18 (2008) $1605-1631$. 
[3] A.-S. Bonnet-Ben Dhia, P. Ciarlet Jr. and C.M. Zwölf, Time harmonic wave diffraction problems in materials with sign-shifting coefficients. J. Comput. Appl. Math. 234 (2010) 1912-1919; Corrigendum J. Comput. Appl. Math. 234 (2010) 2616.

[4] A.-S. Bonnet-Ben Dhia, M. Dauge and K. Ramdani, Analyse spectrale et singularités d'un problème de transmission non coercif. C.R. Acad. Sci. Paris, Ser. I 328 (1999) 717-720.

[5] F. Brezzi and M. Fortin, Mixed and hybrid finite element methods. Springer-Verlag (1991).

[6] L. Chesnel and P. Ciarlet Jr., Compact imbeddings in electromagnetism with interfaces between classical materials and metamaterials. SIAM J. Math. Anal. 43 (2011) 2150-2169.

[7] X. Claeys, Analyse asymptotique et numérique de la diffraction d'ondes par des fils minces. Ph.D. thesis, Université Versailles Saint-Quentin (2008) (in French).

[8] M. Costabel and E. Stephan, A direct boundary integral method for transmission problems. J. Math. Anal. Appl. 106 (1985) $367-413$.

[9] M. Dauge and B. Texier, Problèmes de transmission non coercifs dans des polygones. Technical Report 97-27, Université de Rennes 1, IRMAR, Campus de Beaulieu, 35042 Rennes Cedex, France (1997) http://hal.archives-ouvertes.fr/docs/00/ 56/23/29/PDF/BenjaminT_arxiv.pdf (in French).

[10] L.D. Evans, Partial Differential Equations, Graduate studies in mathematics. Americain Mathematical Society 19 (1998).

[11] P. Fernandes and M. Raffetto, Well posedness and finite element approximability of time-harmonic electromagnetic boundary value problems involving bianisotropic materials and metamaterials. Math. Models Methods Appl. Sci. 19 (2009) $2299-2335$.

[12] V.A. Kozlov, V.G. Maz'ya and J. Rossmann, Elliptic Boundary Value Problems in Domains with Point Singularities, Mathematical Surveys and Monographs. Americain Mathematical Society 52 (1997).

[13] J.-L. Lions and E. Magenes, Problèmes aux limites non homogènes et applications. Dunod (1968).

[14] W. McLean, Strongly elliptic systems and boundary integral equations. Cambridge University Press, Cambridge (2000).

[15] S.A. Nazarov and B.A. Plamenevsky, Elliptic problems in domains with piecewise smooth boundaries, Expositions in Mathematics. De Gruyter 13 (1994).

[16] S. Nicaise and A.M. Sändig, General interface problems-I. Math. Meth. Appl. Sci. 17 (1994) 395-429.

[17] S. Nicaise and A.M. Sändig, General interface problems-II. Math. Meth. Appl. Sci. 17 (1994) 431-450.

[18] S. Nicaise and J. Venel, A posteriori error estimates for a finite element approximation of transmission problems with sign changing coefficients. J. Comput. Appl. Math. 235 (2011) 4272-4282.

[19] G. Oliveri and M. Raffetto, A warning about metamaterials for users of frequency-domain numerical simulators. IEEE Trans. Antennas Propag. 56 (2008) 792-798.

[20] J. Peetre, Another approach to elliptic boundary problems. Commun. Pure Appl. Math. 14 (1961) 711-731.

[21] M. Raffetto, Ill-posed waveguide discontinuity problem involving metamaterials with impedance boundary conditions on the two ports. IET Sci. Meas. Technol. 1 (2007) 232-239.

[22] K. Ramdani, Lignes supraconductrices : analyse mathématique et numérique. Ph.D. thesis, Université Paris 6 (1999) (in French).

[23] A.A. Sukhorukov, I.V. Shadrivov and Y.S. Kivshar, Wave scattering by metamaterial wedges and interfaces. Int. J. Numer. Model. 19 (2006) 105-117.

[24] H. Wallén, H. Kettunen and A. Sihvola, Surface modes of negative-parameter interfaces and the importance of rounding sharp corners. Metamaterials 2 (2008) 113-121.

[25] J. Wloka, Partial Differ. Equ. Cambridge Univ. Press (1987).

[26] C.M. Zwölf, Méthodes variationnelles pour la modélisation des problèmes de transmission d'onde électromagnétique entre diélectrique et méta-matériau. Ph.D. thesis, Université Versailles, Saint-Quentin (2008) (in French). 